\title{
الصحة والإيتيقا: \\ النضج الأخلاقي للممارسة الطبيّة
}

\section{Health and Ethics:}

The Moral Maturity of Medical Practice

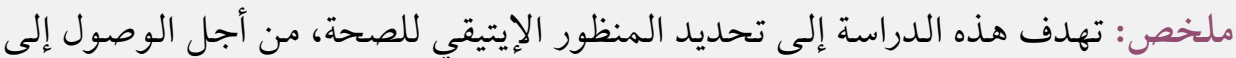

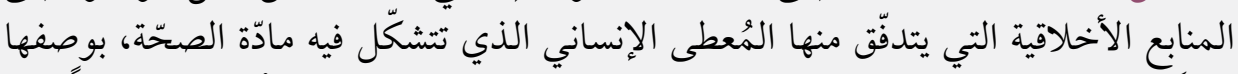

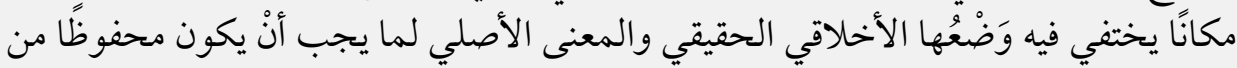

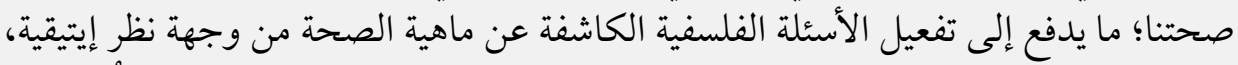

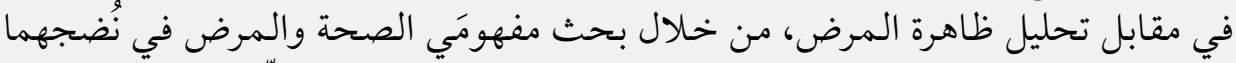

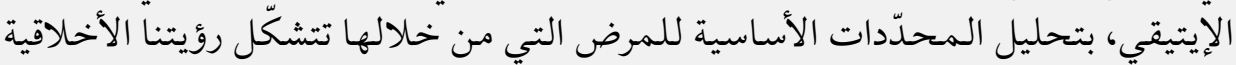

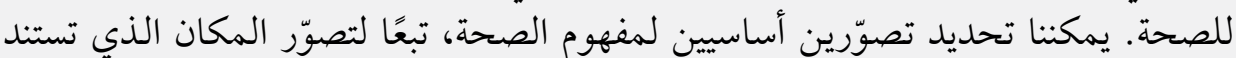

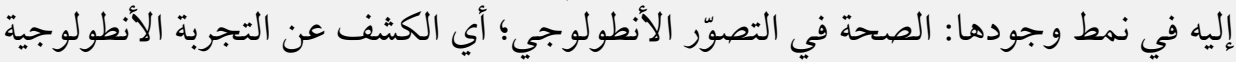

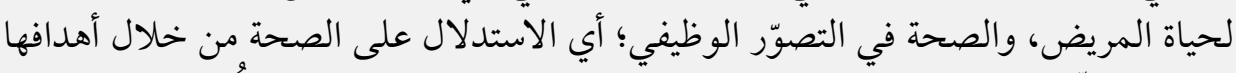

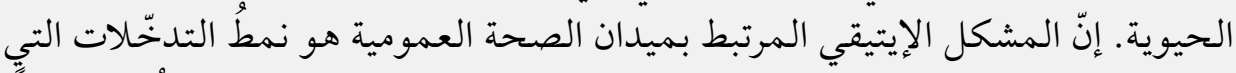

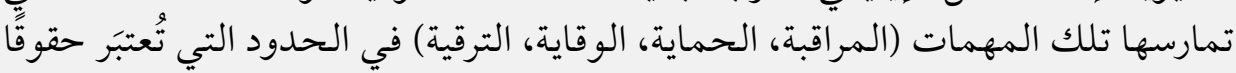

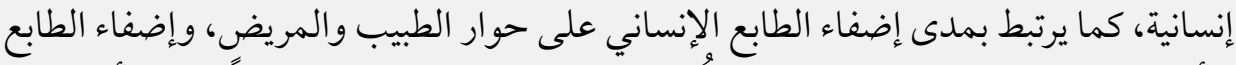

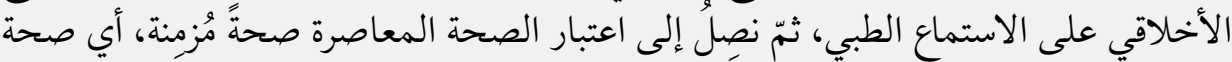

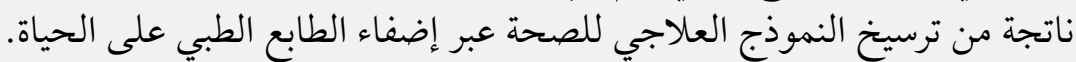

كلمات مفتاحية: الصحة، المرض، الإيتيقا، الممارسة الطبية، الرعاية الصحية، الصحة

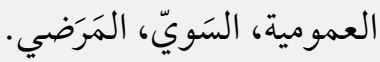

Abstract: The aim of this research is to define an ethical perspective on health in order to arrive at ethical sources from which the human condition springs, a place in which the true moral status and original sense of what in our health must be preserved both disappear. This activates philosophical questions concerning what is exchanged in terms of the ethical point of view in analysis of the phenomenon

Professor of Philosophy, University of Mostaganem, Algeria.

* أستاذ الفلسفة، جامعة مستغانم، الجزائر. 
of disease. We can define two basic conceptions of the concept of health according to where health is based in its conceptual model of existence: health in ontological perception, that is, revealing the ontological experience of a patient's life; and health functionally visualized, that is, inferring health through its vital goals. The ethical problem associated with public health is the type of intervention carried out by these tasks (Surveillance, Protection, Prevention, and Promotion) within what are seen to be human rights boundaries, also related to the extent of humanizing the doctor - patient dialogue and ethical medical listening. We conclude that contemporary health is chronic health, namely health resulting from the application of a therapeutic model for health and medicalizing life.

Keywords: Health, Illness, Ethics, Medical Practice, Health Care, Public Health, Healthy, Pathological.

\section{مقدمة}

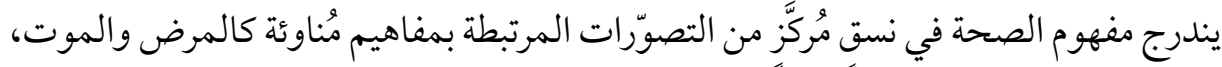

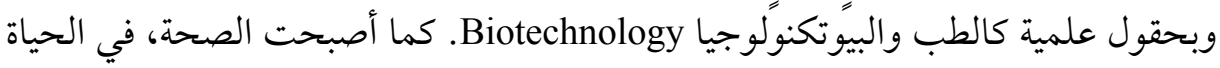

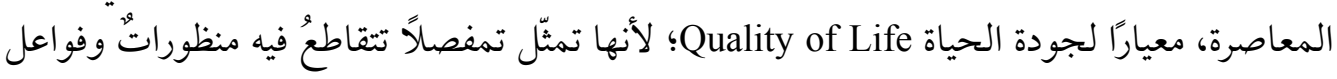

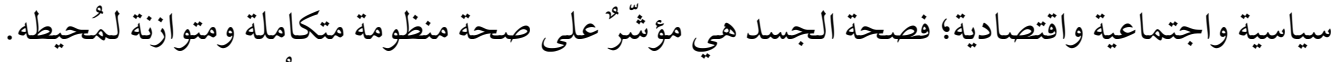

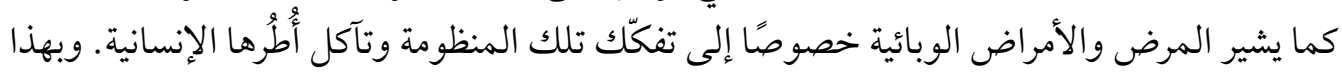

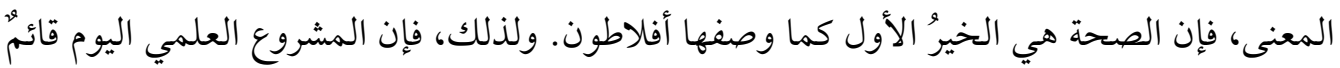

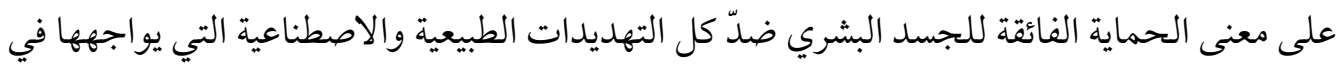

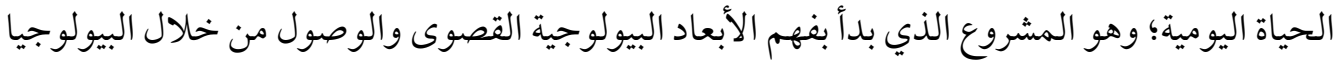

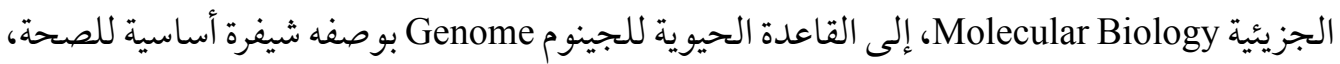
وكذلك فهم الروابط العضوية بين الجسد والمشكلات النفسية من خلال إخضاع الظاهرة النفسية للتشريح الفيسيولوجي العصبي فيما يسمى علم النفس العصبي Neuropsychology.

إن البحث في موضوع الصحة هو عملية تكوين تصوّرات إيتيقية Ethics و إبستيمولوجية، والمؤكّد أن تحصيل الصحة ليس مسألة طبية محضة بل هي مسألة إيتيقية أيضًا مرتبطة بالقيم التي تُرافق ممارسة

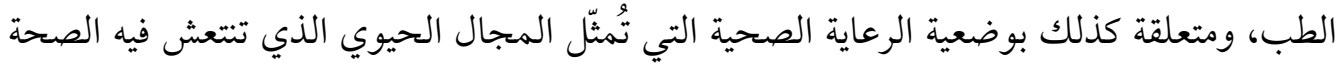

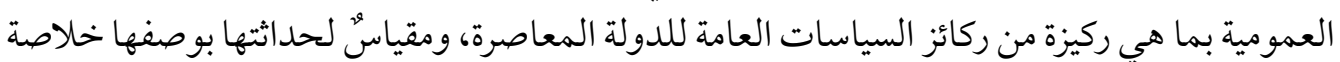
أشكال تطوّر الوعي وأنماط نموّ الثقة وازدهار المسؤولية.

يعني الكشفُ عن المجال الإيتيقي الذي تأخذ فيه الصحةٌ كاملَ فاعليتها الوصولَ إلى المنابع الأخلاقية

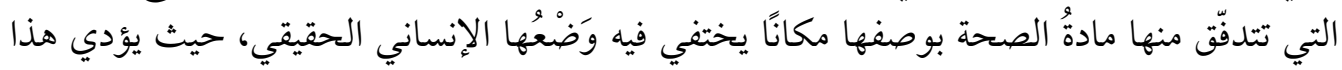

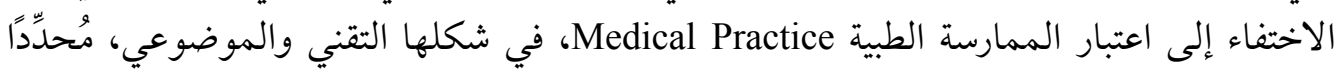

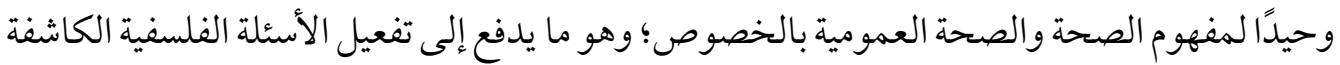
عن ماهية الصحة من وجهة نظر إيتيقية، في مقابل ظاهرة المرض وتحليل القيم المُؤسِّة للصحة 
Care العمومية والعلاقات الإنسانية في الممارسة الطبية، التي تتجسد أساسًا في مفهوم الرعاية الصحية

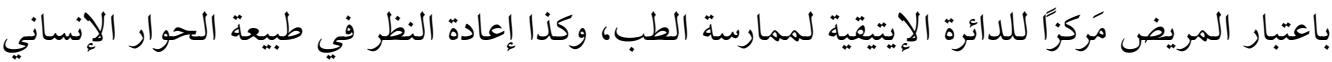

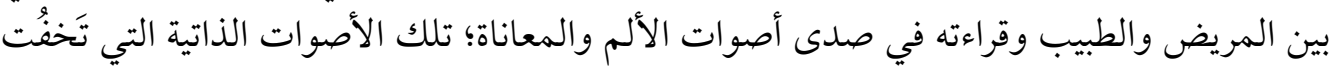

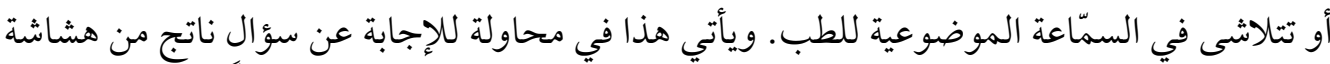

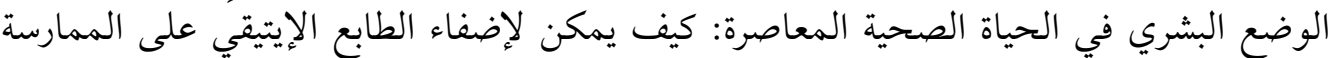

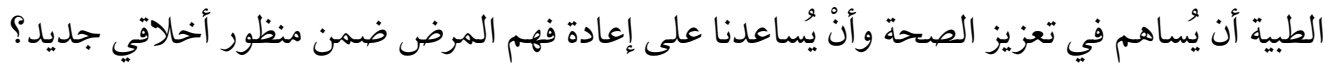

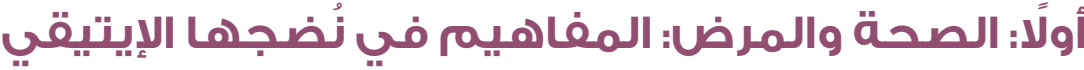

يتأثّر تحديدُنا لماهية الصحة بالمجال العلمي والطبي الذي تتحرّك فيه، وهو المجال الذي يمارس

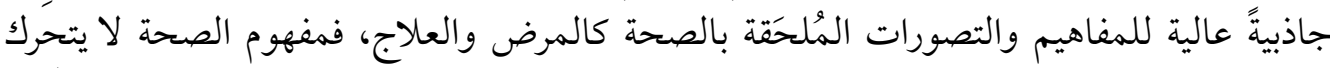

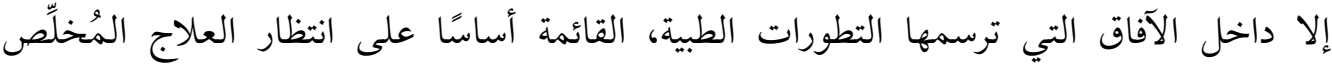
Savior Treatment مكانته في عمليةٍ مستمرة لتوسيع مجاله، وهو ما يحيل عادةً إلى إضفاء الطابع الطبي على الحياة الت Medicalization of Life

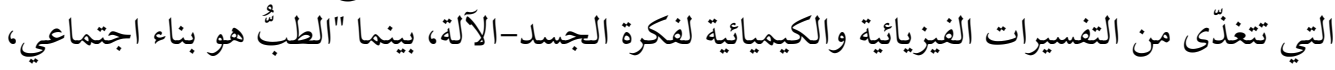
وهذا يعني أن دلالة المفاهيم الطبية مثل الصحة، والمرض، ولثي، ومعايير العلاج هي، من نواح كثيرة، متأثّة

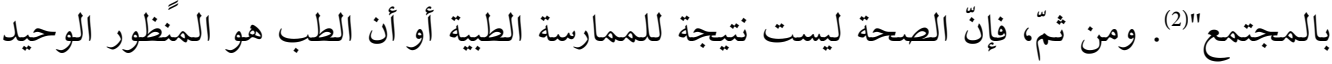

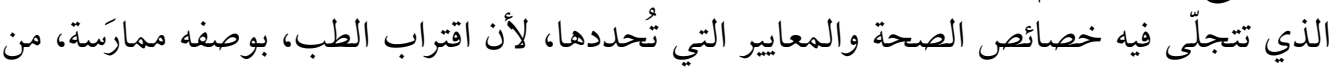

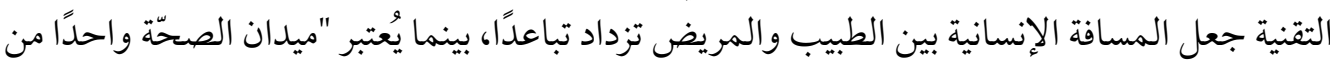

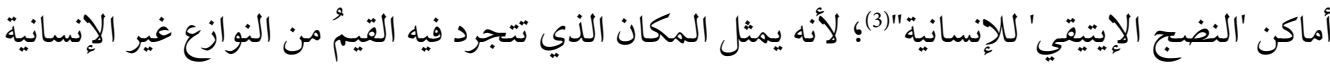

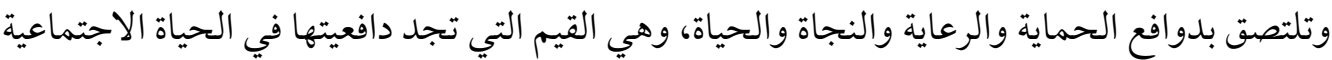

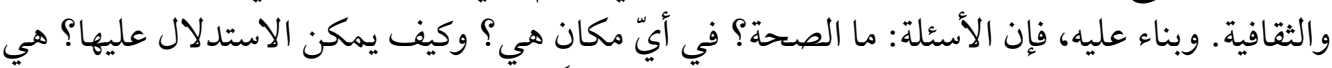

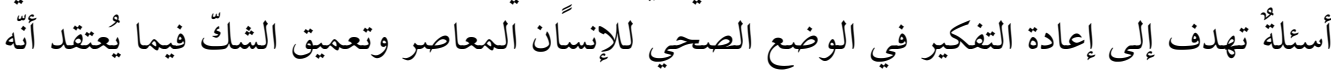
النموذج الأمثل لحياة صحية سليمة.

إن التفكير فلسفيًا في مسألة الصحة يبدأ في بلورة تصور إبستيمولوجي حول السؤال "هل الصحة

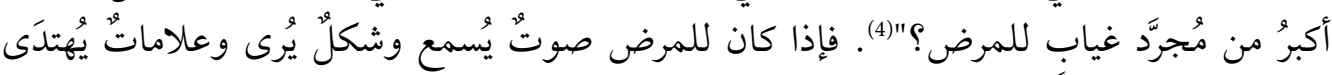

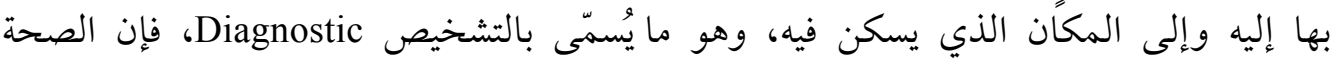

(1) Fredrik Svenaeus, The Hermeneutics of Medicine and the Phenomenology of Health: Steps towards A Philosophy of Medical Practice (Berlin/ Dordrecht: Springer Science \& Business Media, 2000), p. 56.

(2) Basile Ekanga, Les fondements éthiques de la Bioéthique (Bloomington: iUniverse LLC, 2013), p. 22.

(3) Bruno Cadoré, L'expérience bioéthique de la responsabilité (Paris: Artel; Montréal: Fides, 1994), p. 64.

(4) Pierre-Olivier Méthot, "Les concepts de santé et de maladie en histoire et en philosophie de la médecine," Revue Phares, vol. XVI, Université Laval (2016), p. 9. 


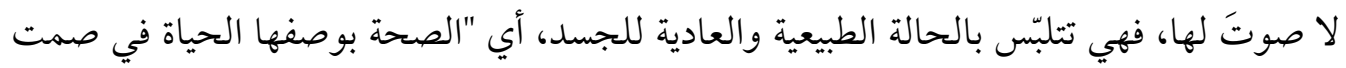

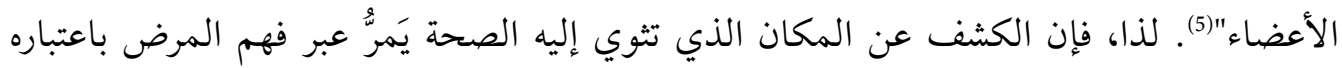

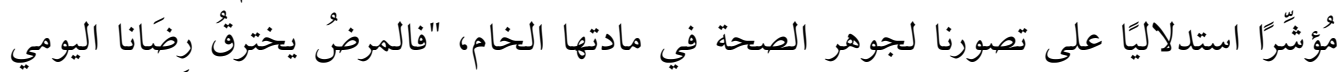

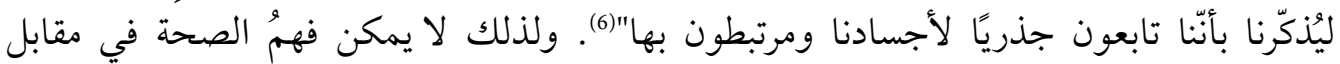

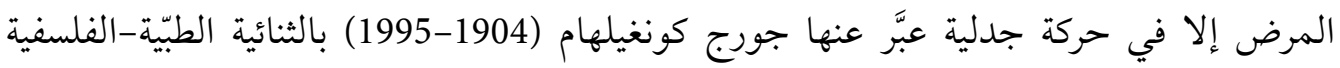

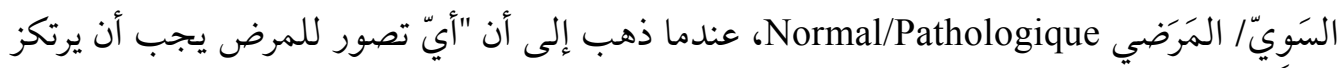

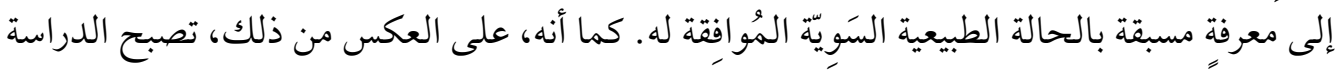

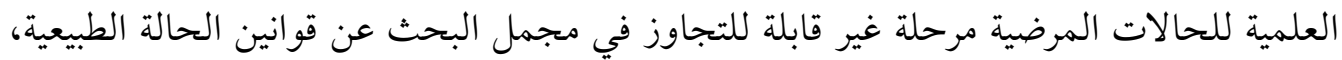

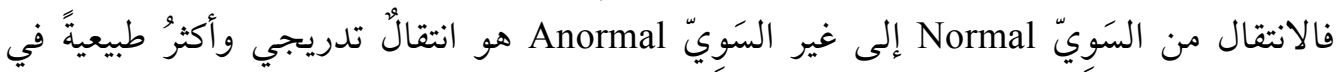

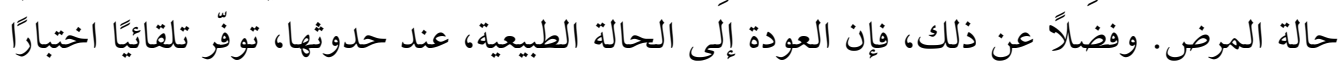

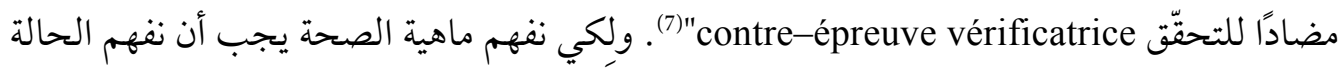

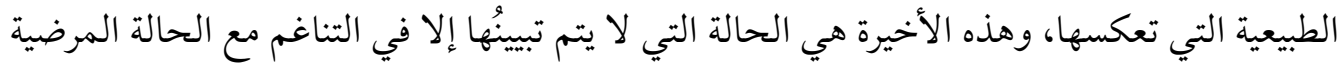

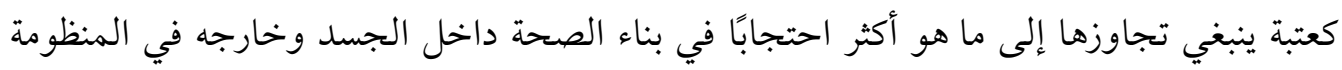
الاجتماعية والثقافية المحيطة بالجسد نفسه.

لماذا يجب أن نبحث فيما وراء الظاهر من الصحة والمرض، أيْ في تقديم مقاربة تأويلية لهما؟ لأن

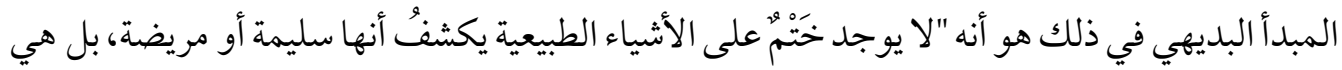

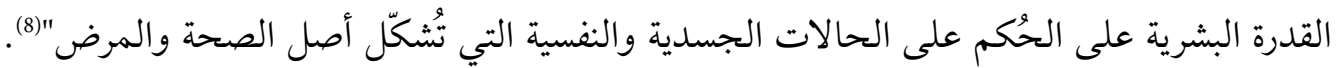

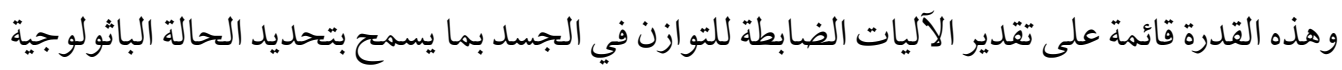

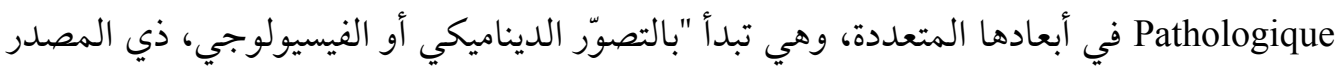

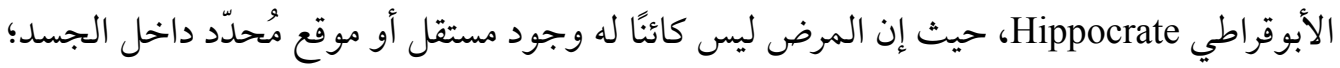

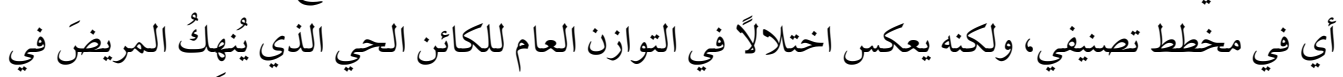

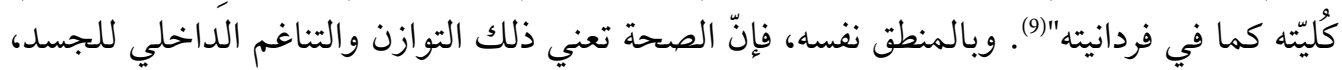

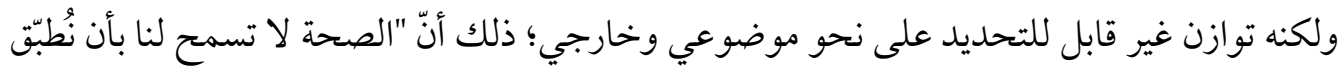

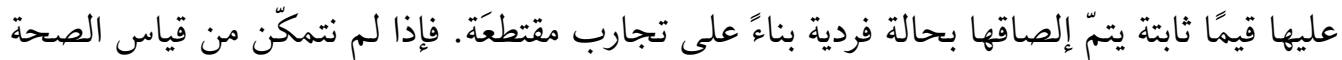

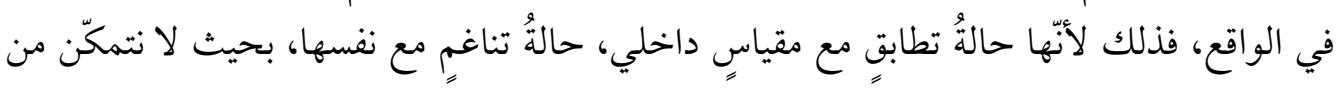

(5) Cadoré, p. 60.

(6) Carol Taylor \& Roberto Dell'oro, Health and Human Flourishing Religion, Medicine and Moral Anthropology (Washington, DC: Georgetown University Press, 2006), pp. 120-121.

(7) Georges Canguilhem, Le normal et le Pathologique, $12^{\mathrm{eme} e}$ é (Paris: Presses Universitaires de France, 2013), p. 27.

(8) Antje Gimmler, Christian Lenk \& Gerhard Aumuller, Health and Quality of Life: Philosophical, Medical and Cultural Aspects (Homburg/ London/ Munster: LIT, 2002), p. 9.

(9) Méthot, p. 12. 
مراقبتها من الخارج بالكامل"(10). فالصحة، إذًا، انكشاف ذاتي لما يُحقّق التناغم الداخلي للجسد، وهو التناغم الذي تشترك فيه التمثلات الاجتماعية والانخراط الثقافي للجسد في منظومة القيم التي تدفعه إلى العيش وفق نمط يتصوره "صحيًا" وهذا كلّه يضع الصحة داخل بؤرة توتر إيتيقية تعمل على توليد الإنساني من الطبيعي.

إنّ معرفتنا بالمرض، المتشكّلة أساسًا من خلال تطور العلاجات الطبية وابتكار العيادة بوصفها مؤسسة ذات سلطة واسعة في المجتمع المعاصر، هي معرفة قائمة على التمييز بين المرض بوضئ بوصفه مجموعة

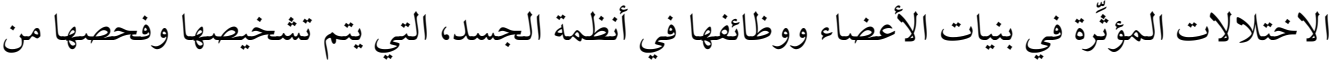

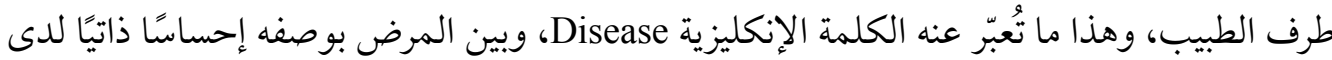
المريض، كإحساسه بالألم وما يُصاحبه من أعراض تدله على على على وجود مشكلة صحية، وهذا ما تُعبّر عنه

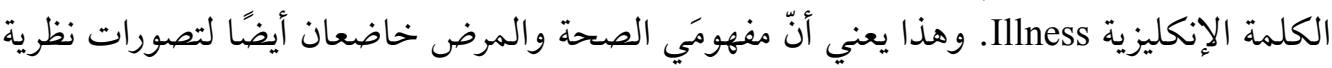

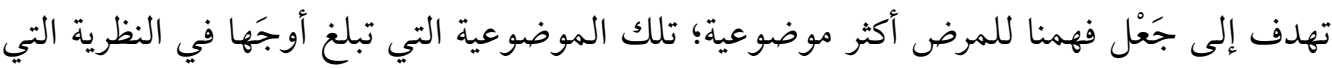
قدّمها الفيلسوف الأميركي كريستوفر بورس (1977-1997) حول الإحصائية الحيوية Biostatistics، وفي المثال الذي قدّمه حول الصحة العقلية، حيث "تخضع الأحكام حول الصحة لتقييمٍ من طرف

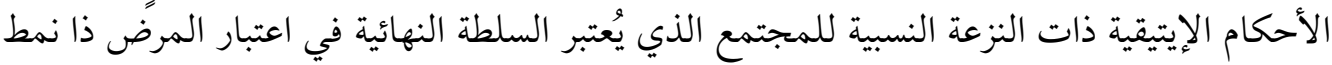

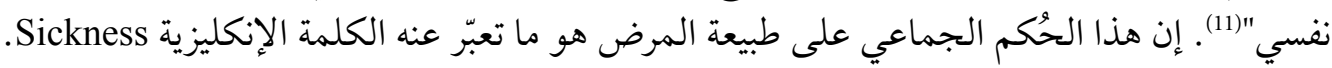

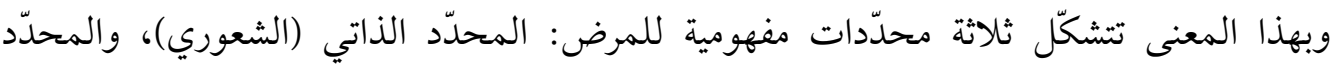
الموضوعي (الطبي)، والمحدّد الجمعي.

1. محددات مغهومية للمرض

$$
\text { أ. المحلّد الذاتي للمرض }
$$

المرض الذي تعبّر عنه كلمة Illness هو عيشه بوصفه تجربة داخلية للإحساس بالألم، وملاحظة

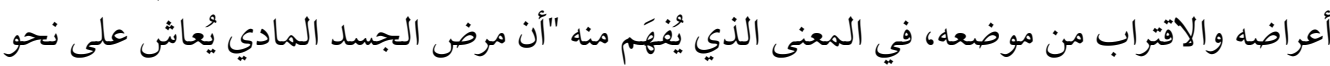

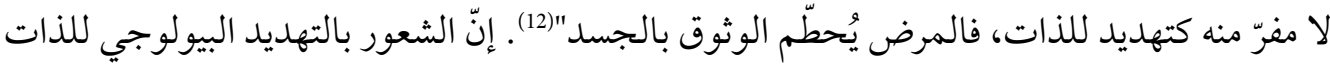

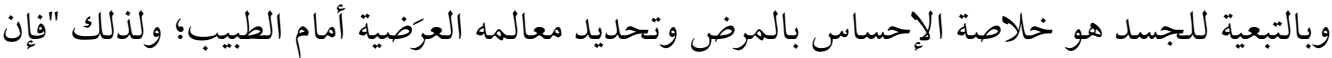

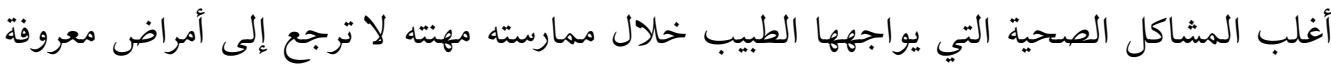
Diseases

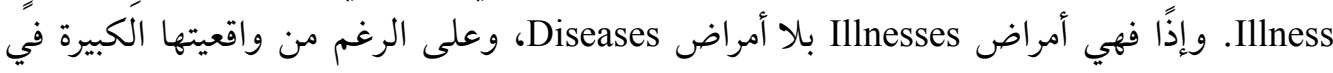

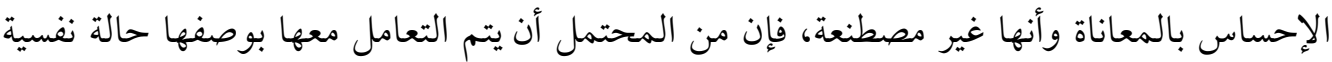

(10) Hans-Georg Gadamer, Philosophie de la santé, Marianne Dautrey (trad.) (Paris: Grasset, 1998), p. 117.

(11) Christopher Boorse, "On the Distinction between Disease and Illness," in: Christopher Boorse et al., Meaning and Medicine: A Reader in the Philosophy of Health Care (New York: Routledge, 1999), p. 17.

(12) Taylor \& Dell'oro, p. 121. 
واجتماعية للمريض (13)، فتكون الذاتُ مركزًا لتحديد المريض وطريقة عَرْضه وإبلاغه للطبيب، وهنا

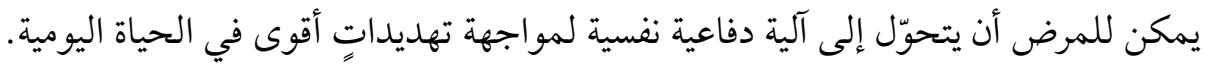

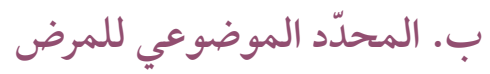

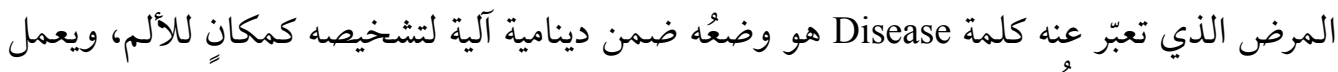

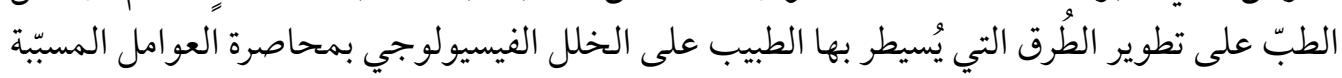

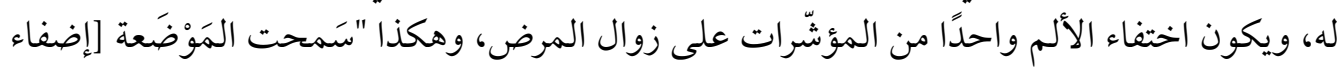

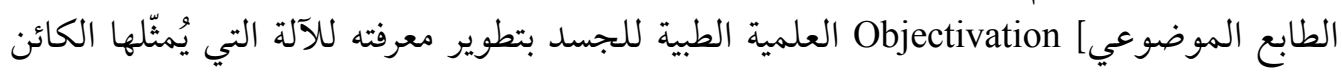

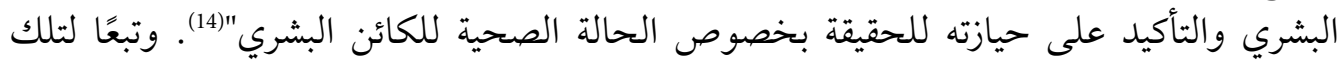

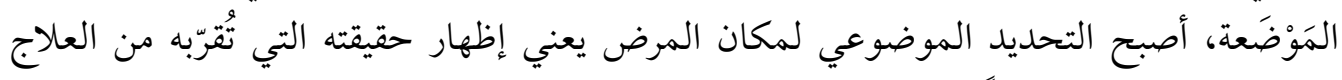

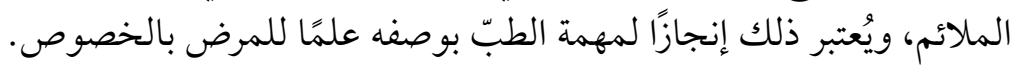

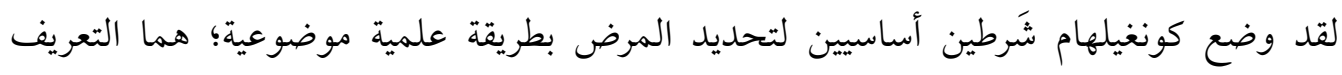

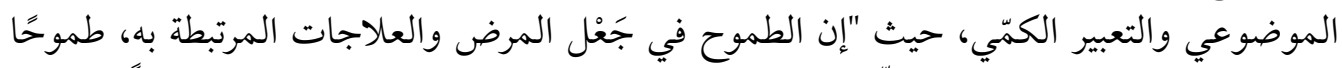

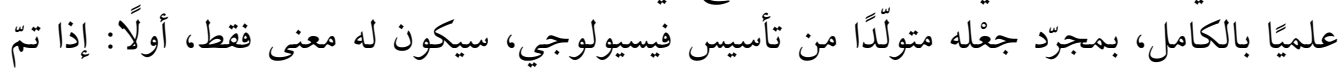

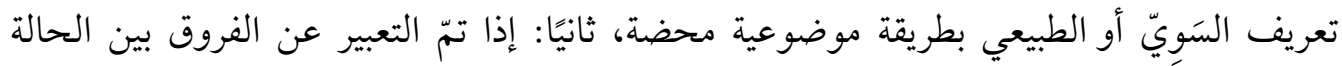

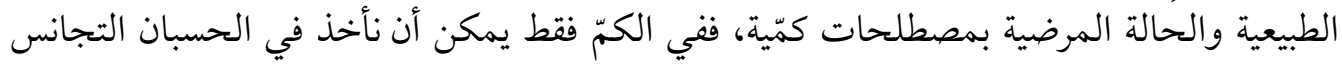

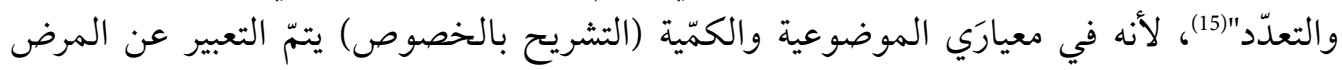

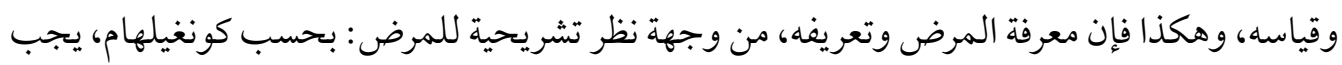

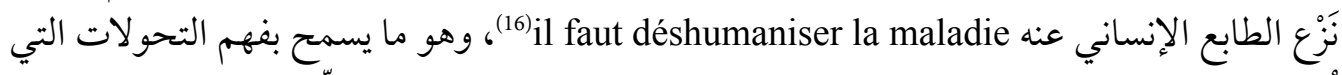

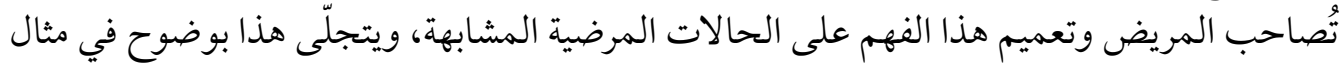

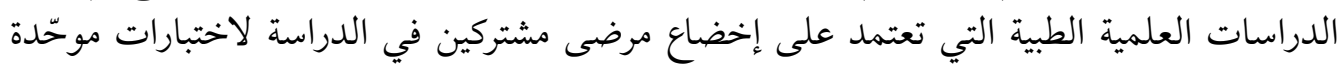
لاستخراج نتائج ذات طابع عام.

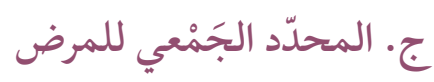

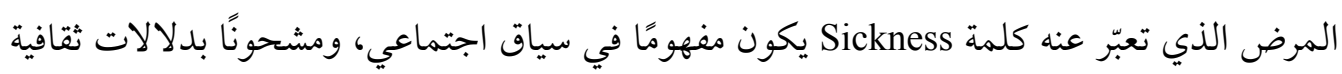

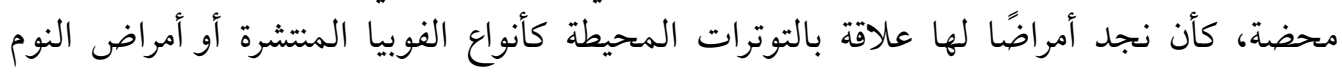

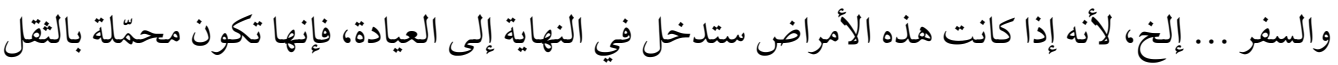

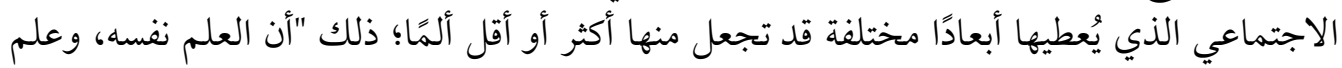

(13) Svenaeus, p. 40.

(14) Pierre Boitte, Éthique, justice et santé: Allocation des ressources en soins dans une population vieillissante (Paris: Artel; Montréal: Fides, 1995), p. 20.

(15) Canguilhem, p. 33.

(16) Lucie Rey, Qu'est-ce que la douleur? Lecture de René Leriche (Paris: Éditions L'Harmattan, 2010), p. 9. 


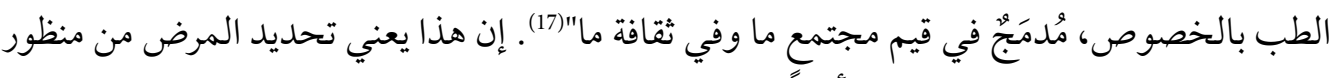

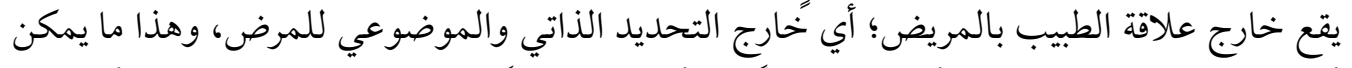

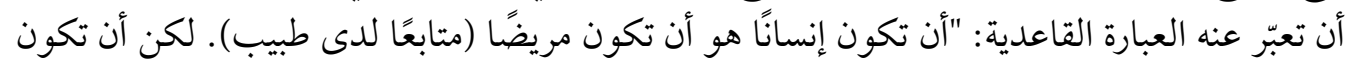

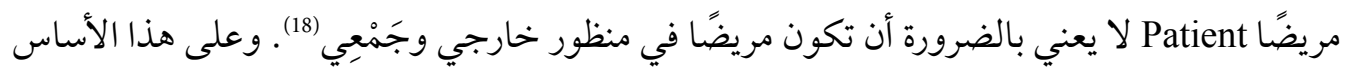

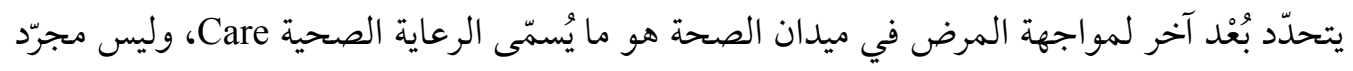
العلاج في مفهومه داخل منظومة الممارسة الطبية.

\section{2. - تصوران أساسيان للصحة}

كما يمكننا تحديد تصورين أساسيين لمفهوم الصحة تبعًا لتصور المكان الذي تستند إليه الصحة في

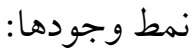

$$
\text { أ. أ. الصحة في التصور الأنطولوجي }
$$

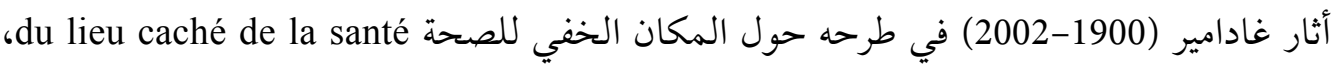

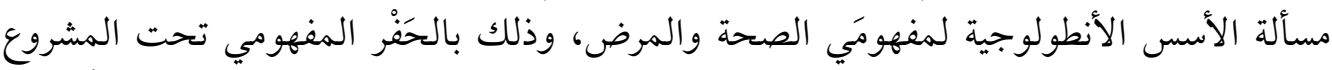

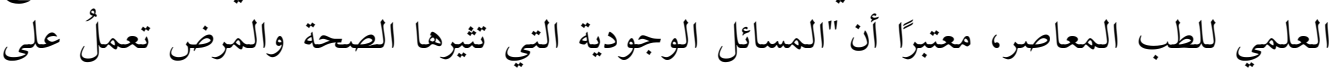

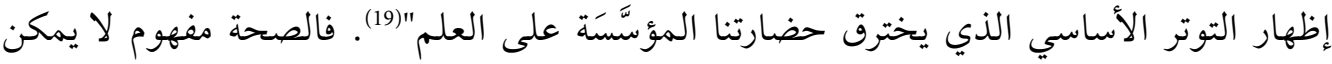

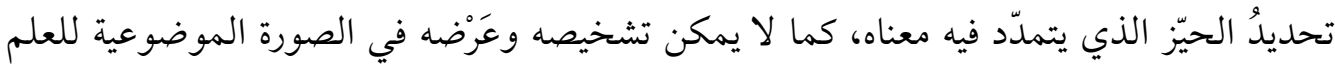

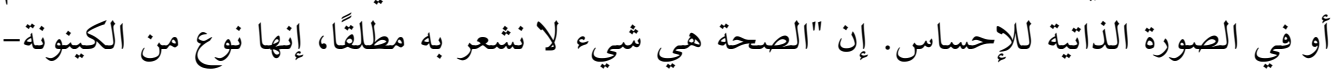

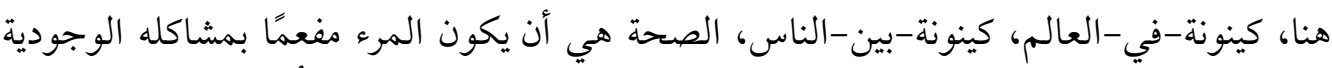

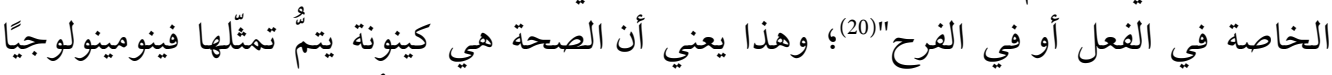

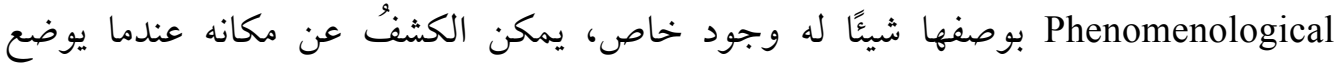

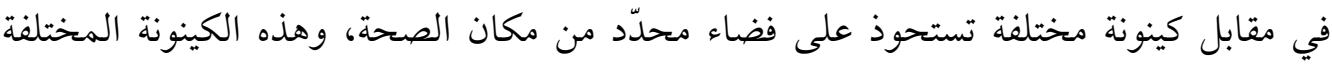

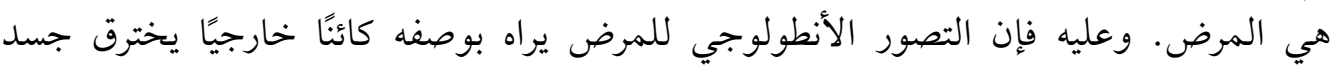

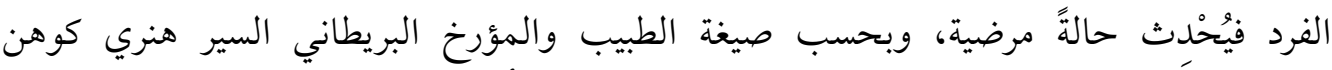
Sir Henry Cohen

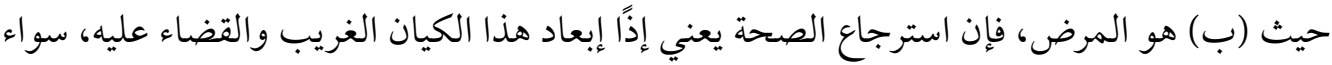

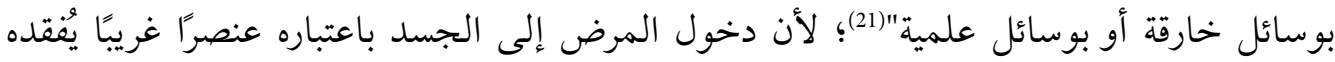

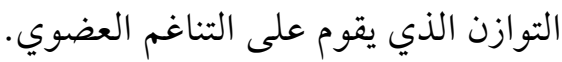

(17) Ekanga, p. 22.

(18) Taylor \& Dell'oro, p. 60.

(19) Gadamer, p. 114.

(20) Ibid., p. 122.

(21) Méthot, p. 13. 


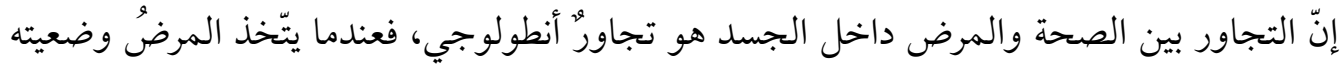

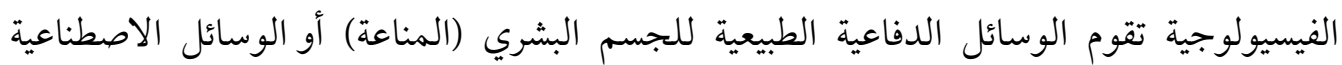

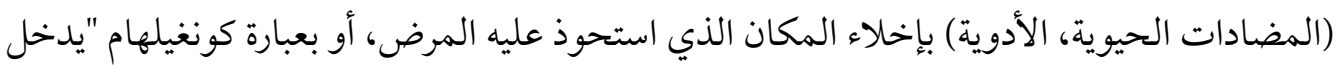

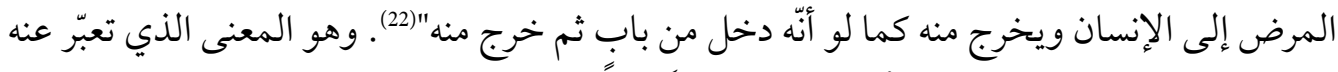

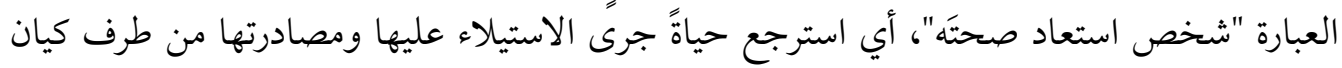

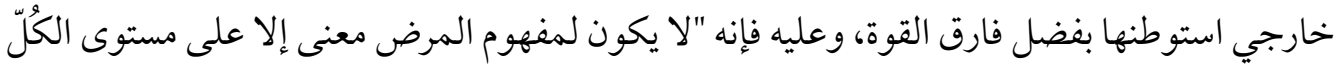

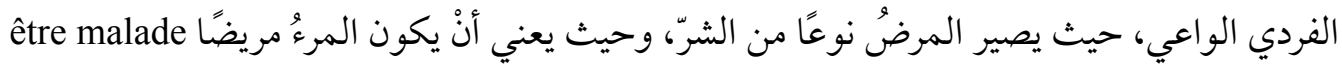

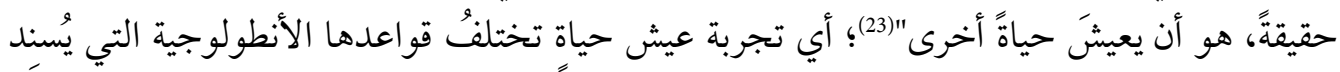

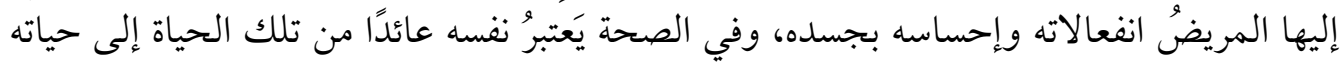
الأصلية والخاصة.

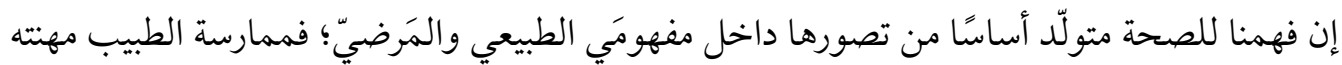

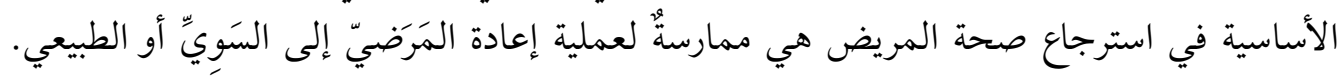

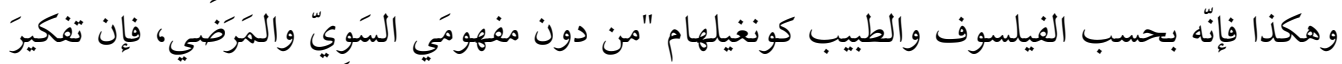

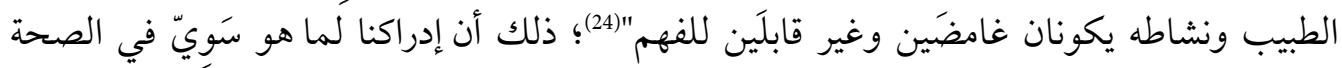

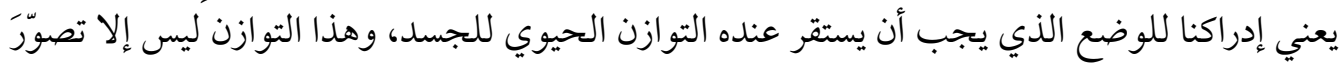

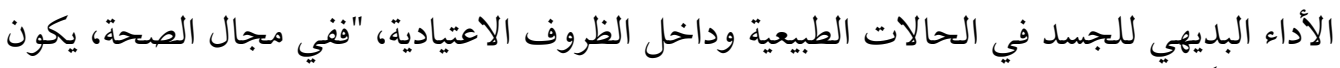

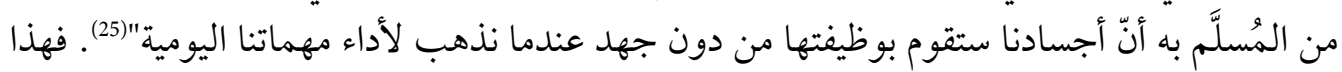

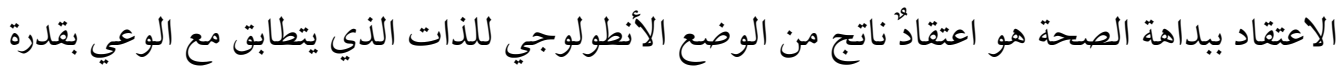

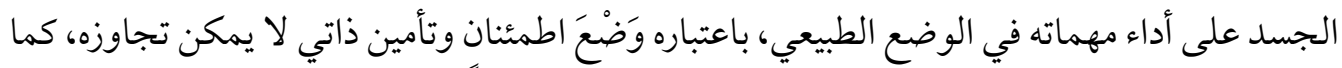

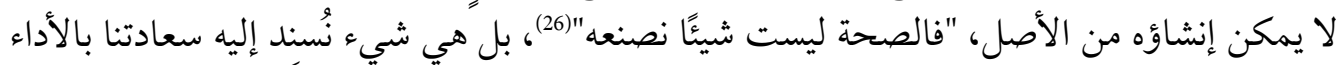

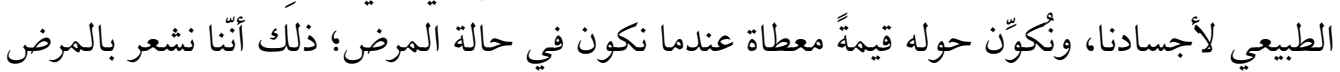

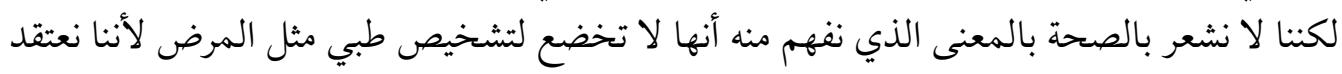

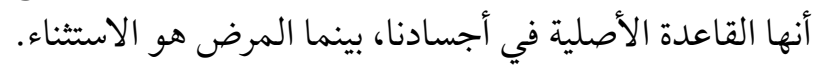

$$
\text { ب. إذا الصحة في التصور الوظيفي }
$$

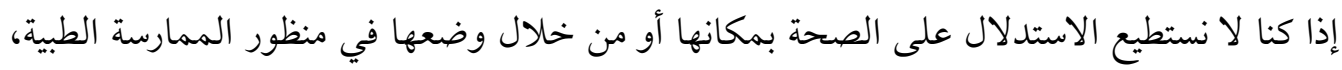

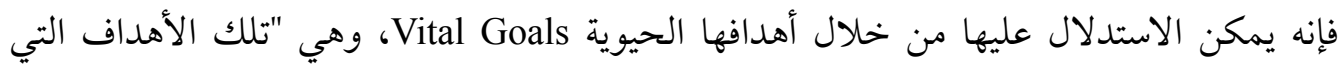

(22) Canguilhem, p. 13.

(23) Rey, p. 9.

(24) Méthot, p. 16.

(25) Taylor \& Dell'oro, p. 121.

(26) Gadamer, p. 7. 
يحتاج الفردٌ إلى إنجازها ليكون سعيدًا بالحد الأدنى لمعنى السعادة"(27). وبناء على هذا المفهوم، قدّم الفيلسوف السويدي لينارت نوردنفلت Lennart Nordenfelt تعريفًا للصحة بأنها "قابلية تحقيت هليق واحد من الأهداف الحيوية في ظل الظروف الطبيعية"(28). وهذا التعريف قائم على الربط بين الصحة

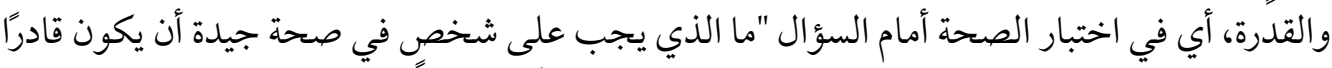

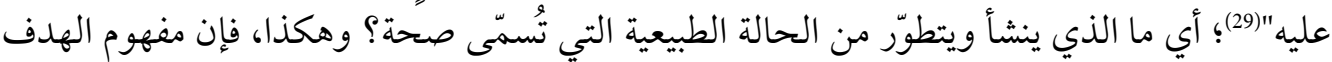

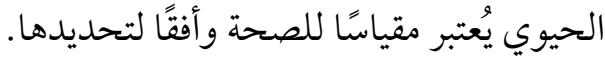

\section{ثُنانيًا: إيتيقيقا الصحمة العمومية والرعاية الصحية}

تحوّل موضوع الصحة في الدولة الحديثة من كونه مسألة فردية مرتبطة بالعلاج إلى قضية ذات طابع

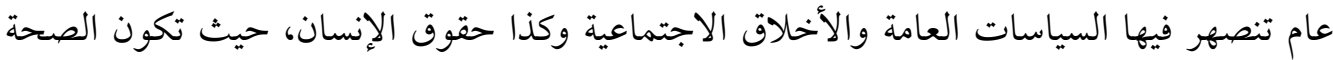
أمام سؤالين أساسيين: كيف يمكن توفير الرعاية الصحية للمجتمع من دون التأثير في القيم التي يقوم

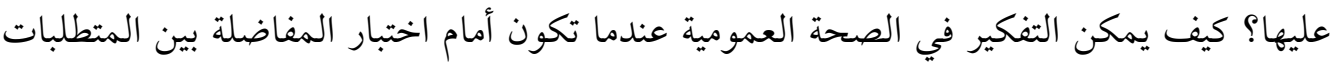
الموضوعية للممارسة الطبية التي تقوم على الإكراه العلاجي وممارسة السلطة المطلقة، فيما يُعتقد أنه

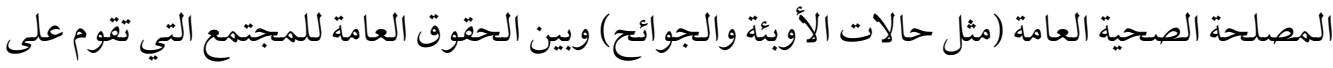

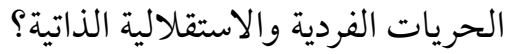

يمكن البدء بالتعريف الأشهر للصحة العمومية بما هي "علم وفنّ مَنْع المرض وإطالة الحياة، وترقية

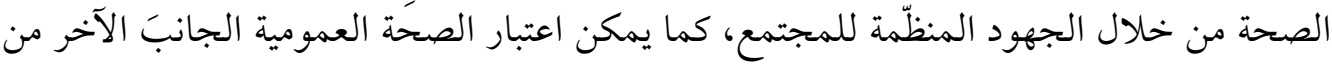

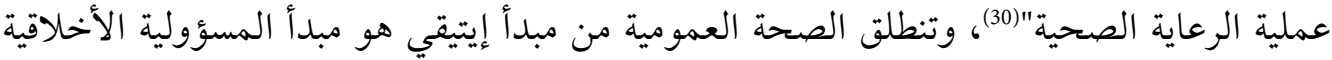

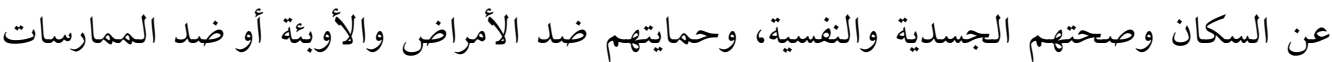

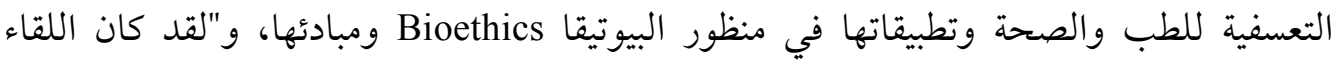

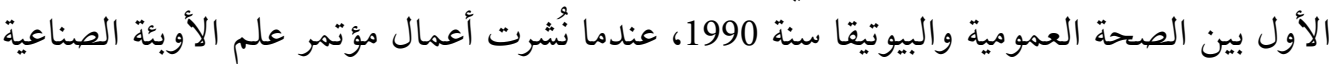

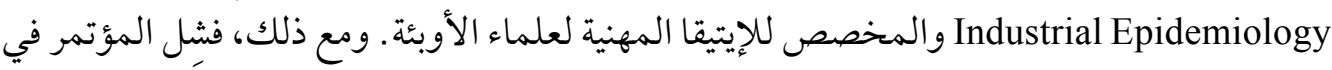
خلق اهتمام مستمر بإيتيقا الصحة العمومية؛ إذ في ذروة البيوتيقا الإكلينيكية Clinical Bioethics،

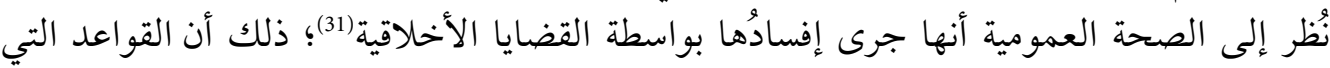

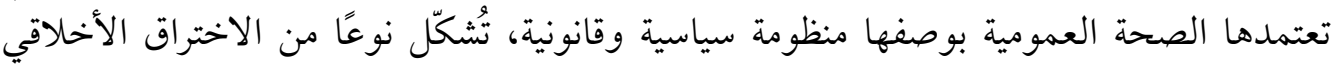

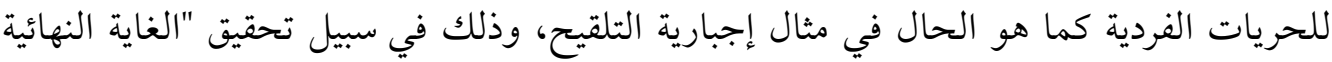

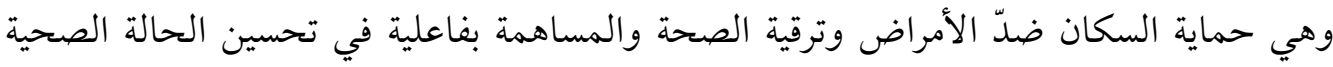

(27) Svenaeus, p. 73

(28) Ibid., p. 69.

(29) Méthot, p. 27.

(30) Angus Dawson (ed.), The Philosophy of Public Health (London/ New York: Routledge, 2016), p. 52.

(31) Miguel Kottow, From Justice to Protection: A Proposal for Public Health Bioethics (New York: Springer, 2012), p. 34 . 
وكذا تقليص نسبة الوفاة ونسبة المرض morbidité للسكان"(32). ولتحقيق تلك الغاية، تشتغل

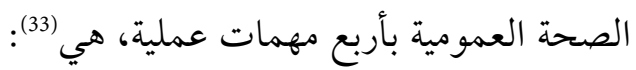

1. المراقبة Surveillance: هي عملية متواصلة لتقدير الحالة الصحية ورفاه السكان ومحدّداتها. 2. Protection تُحيل إلى تَدُُّل مسؤولي الصحة العمومية لحماية الأفراد والجماعات والسكان في حالة تهديد حقيقي للصحة.

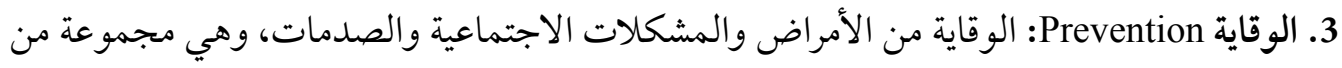

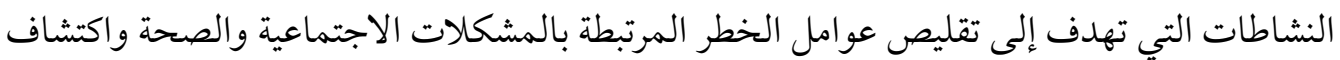
العلامات المبكرة Hâtifs للمشكلات من أجل التحل التحكّم فيها. 4. الترقية Promotion: ترقية الصحة وتتعلق بمجموعة النشاطات التي تدعم فعل الأفراد والمجموعات

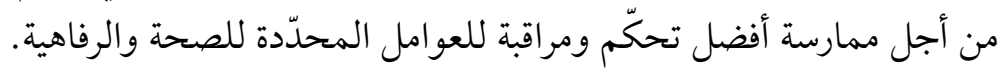

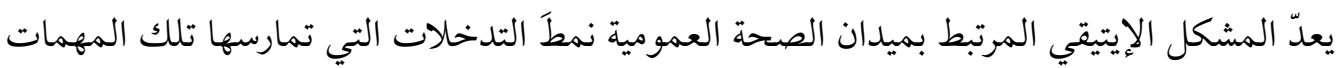

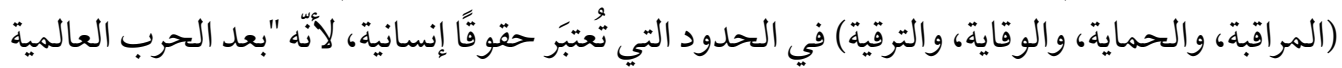

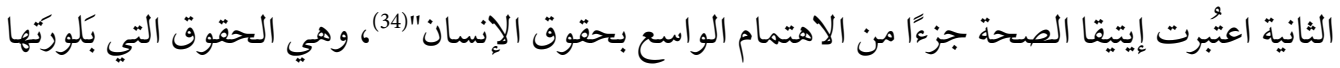

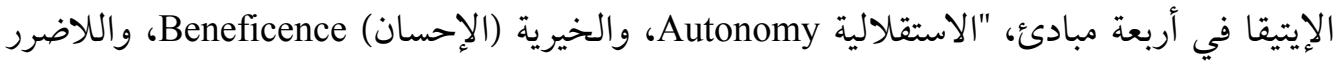
Non-Maleficence

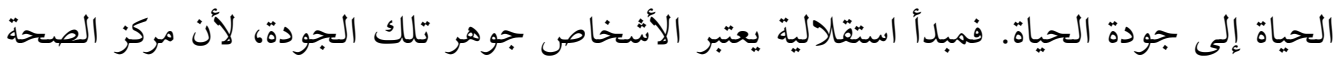

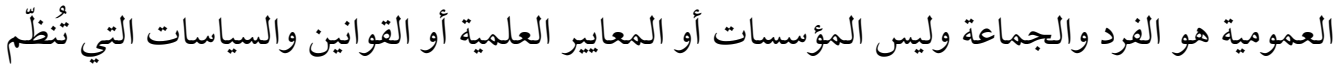

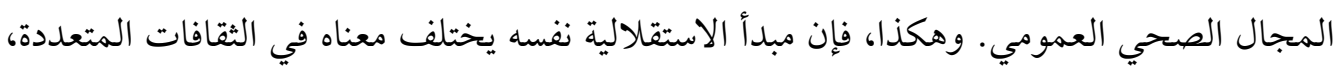

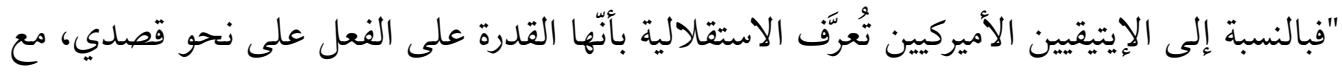

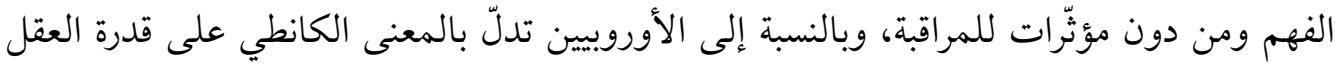

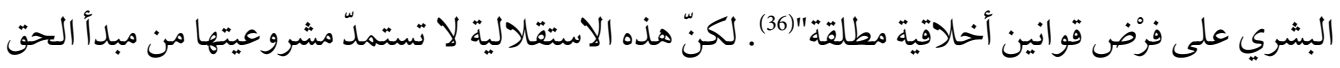

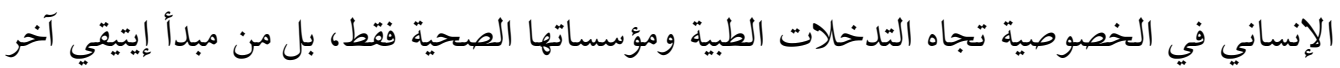

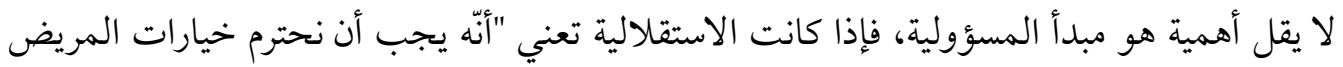

(32) Raymond Massé, Éthique et santé publique: Enjeux, valeurs et normativité (Québec: Les presses de l'université Laval, 2003), p. 115.

(33) Ibid., p. 6.

(34) Frank Lewins, Bioethics for Health Professionals: An Introduction and Critical Approach (Melbourne: Macmillan Education Australia, 1996), p. 10.

(35) Nancy S. Jecker, Albert R. Jansen \& Robert A. Pearlman, Bioethics: An Introduction to the History, Methods, and Practice (Sudbury: Jones and Bartlett Publishers, 2007), p. 103.

(36) Massé, p. 146. 
القادر والمؤهّل لأخذ قرارات تخصّه"(37) فإنّه، في المقابل، يجب على المريض أن يكون مسؤولًا عن

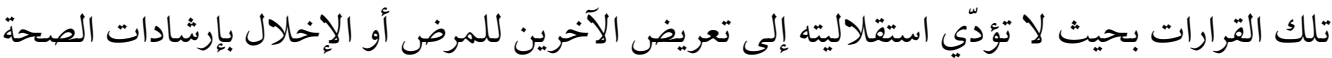

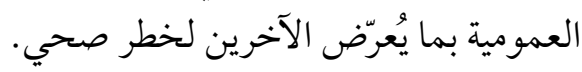

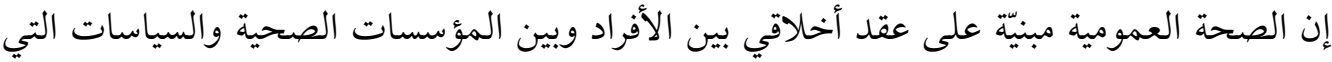

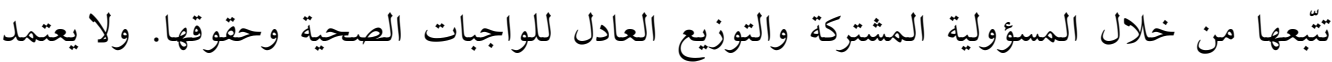

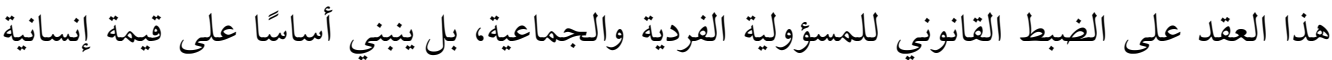

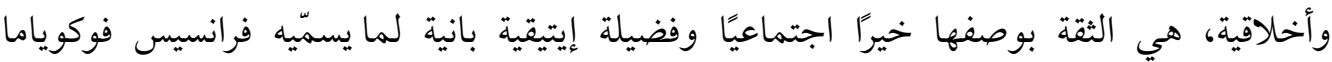

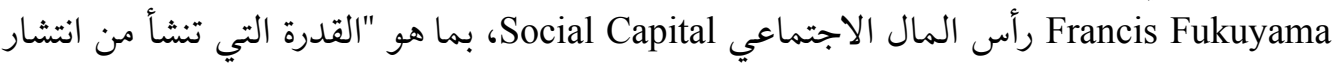
الثقة في المجتمع" (38). ومن ثمّ، فإن الصحة العمومية تأخذ قوتها الاجتماعية ومشروعيتها الأخلاقية

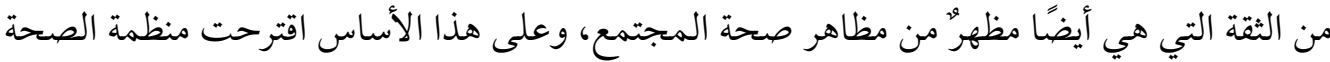

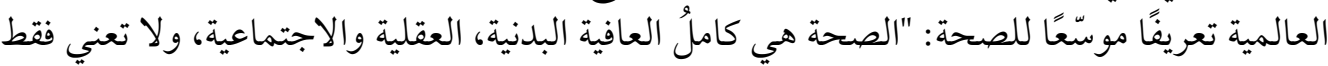

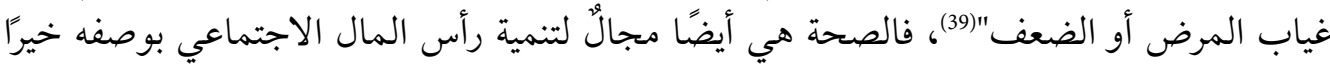
أخلاقيًا؛ ذلك أن "الصحة تؤدي اليوم دور المُحفّز على تحليل وبناء، أو ترقية، الرابطة الاجتماعية" (40)

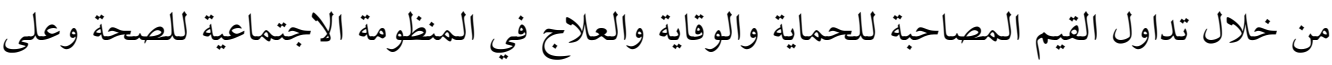
رأسها المسؤولية الفردية والمؤسساتية.

لا ترتبط الصحة العمومية بالترقية التكنولوجية لوسائل العلاج وأدوات التطبيب أو تشييد ترسانتها

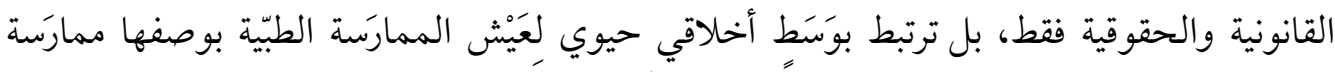

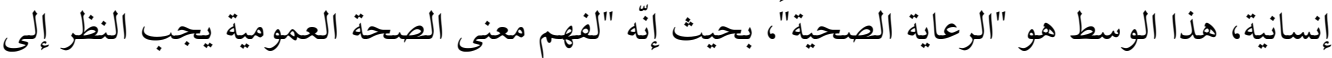

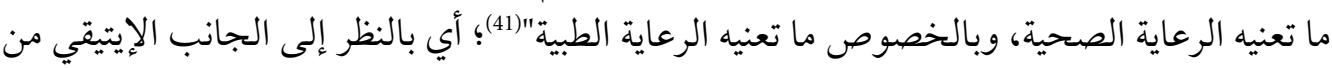

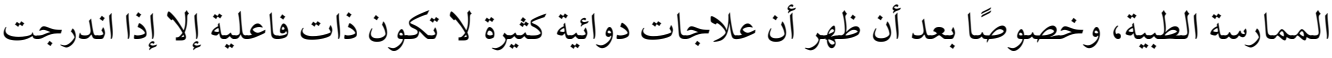
ضمن تأهيل نفسي وسلوكي للمريض في منظور إنساني شامل، وتعني الرعاية الصحية "نشاطًا تقنيًا

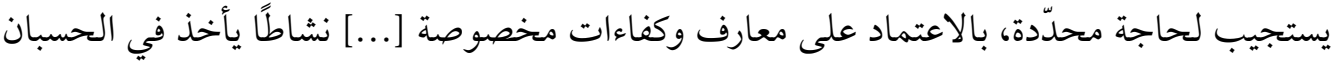

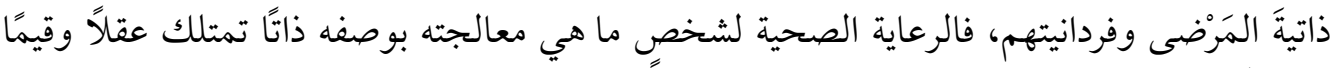

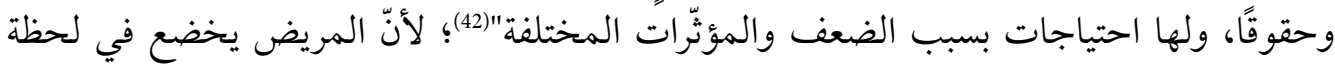

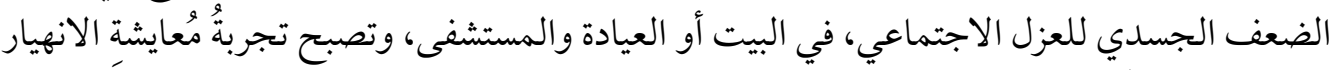

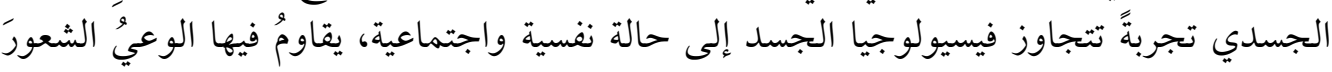

(37) Ekanga, p. 10.

(38) Dawson (ed.), p. 40.

(39) Méthot, p. 17.

(40) Cadoré, p. 68.

(41) Dawson (ed.), p. 52.

(42) Céline Lefèvre, "De la philosophie de la médecine de Georges Canguilhem à la philosophie du soin médical," Revue de Métaphysique et de morale, vol. 2, no. 82 (2014), pp. 199-200. 


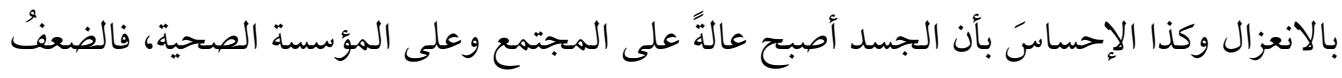

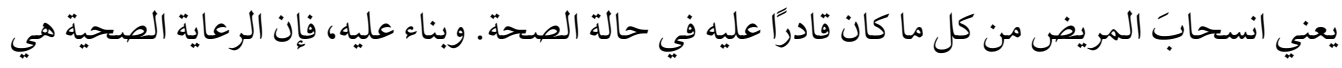

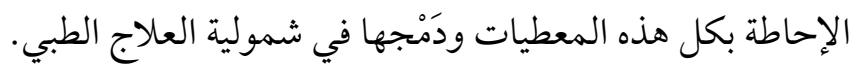

لا يخلو مفهوم الرعاية الصحية من جدل إيتيقي وسياسي على السواء، "فالبعض اعتبَ أن إيتيقا الرعاية

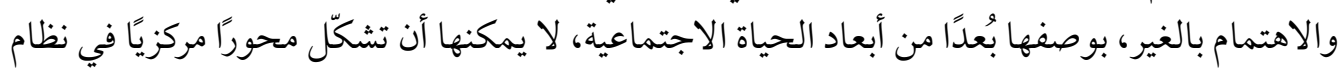

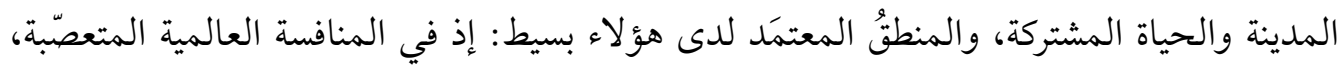

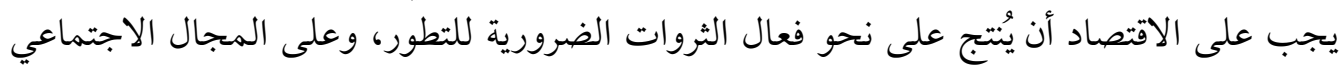

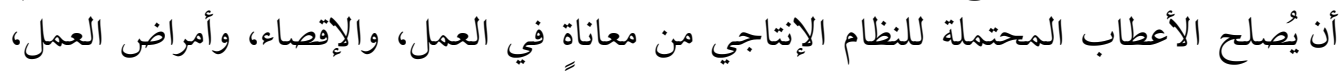

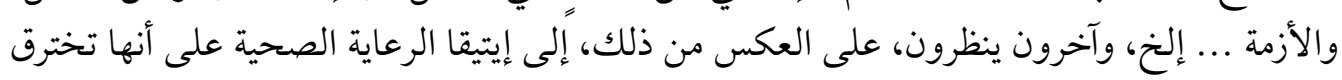

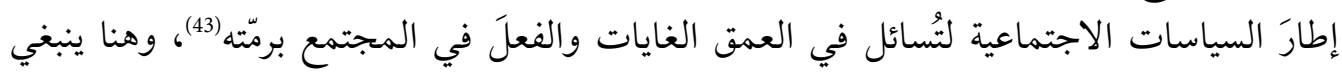

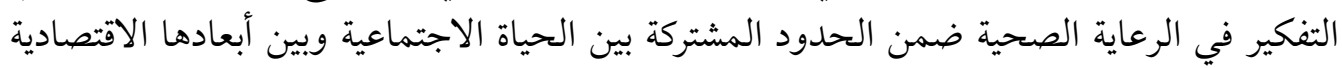

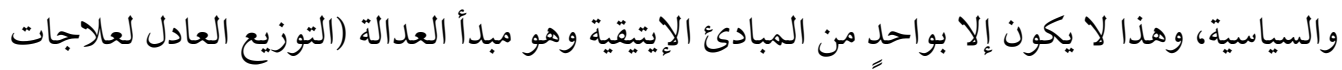

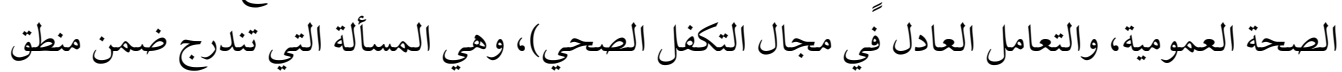

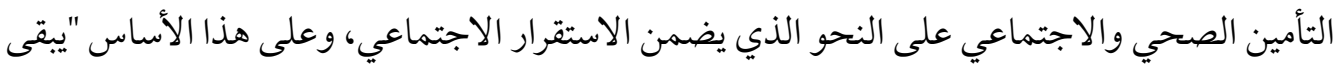

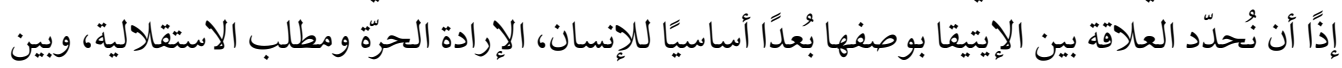

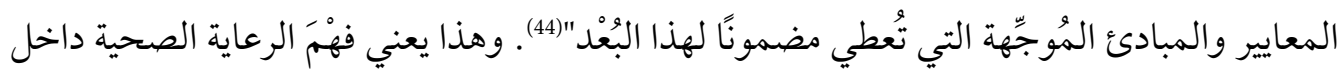

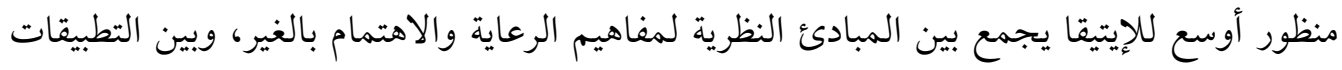

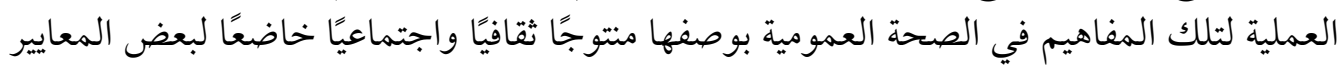

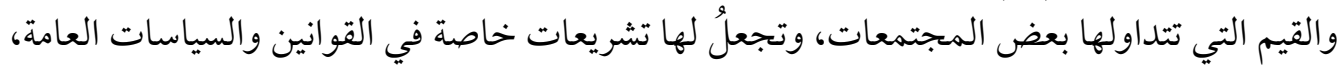

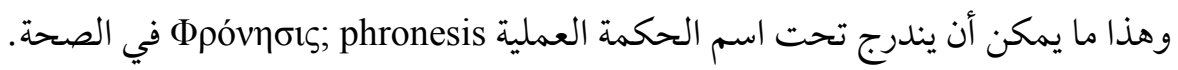

\section{ثُالثًا: حوار الطبيب والمريض: السمّاعة الموضوعية

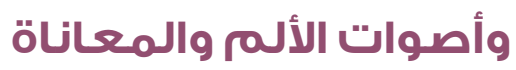

في تراثٍ يمتدّ من الفكر اليوناني القديم الذي اعتَبر أن صحة الجسد تتطلب صحةً نفسية فاضلة

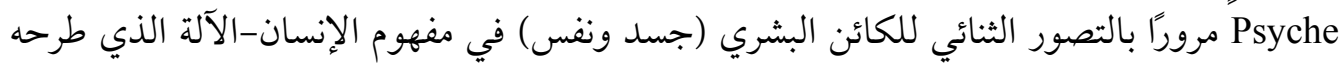

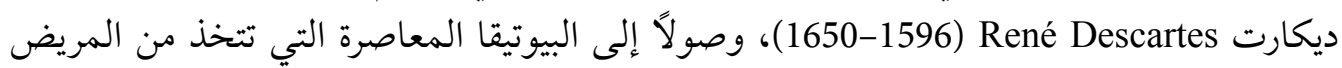

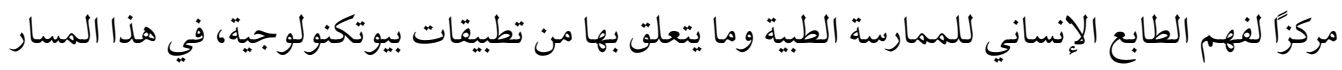

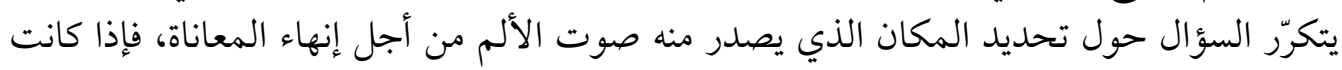

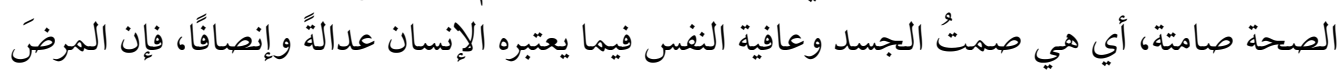

(43) Paulette Guinchaud \& Jean François Petit, Une société de soins: Santé, travail, philosophie, politique (Paris: Les éditions de 1'Atelier; Les éditions ouvrières, 2011), p. 5.

(44) Boitte, p. 84. 


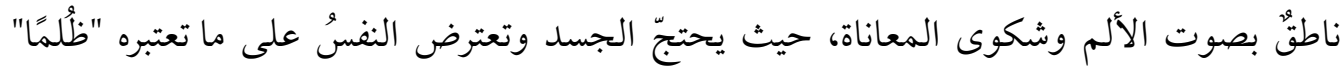

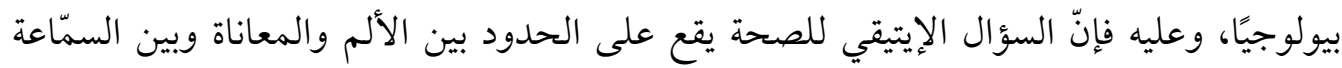

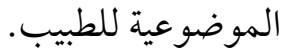

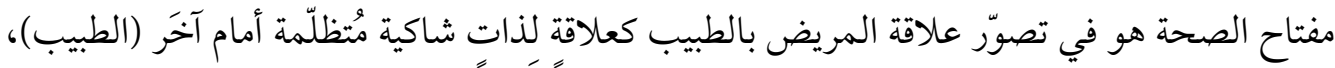

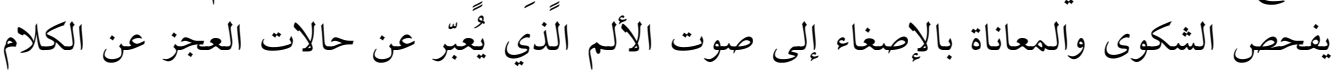

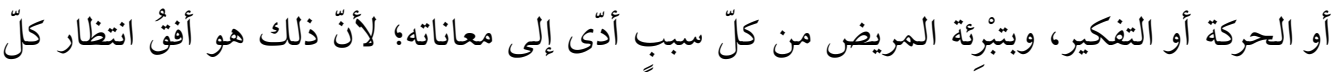

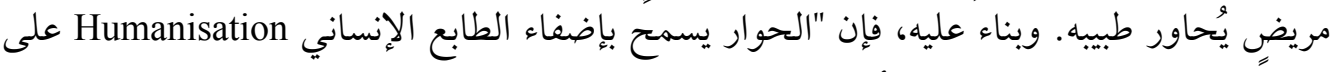

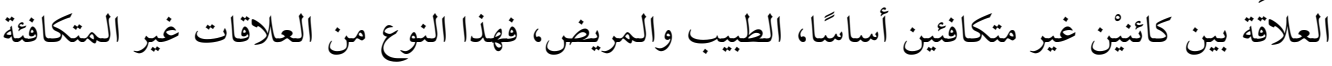

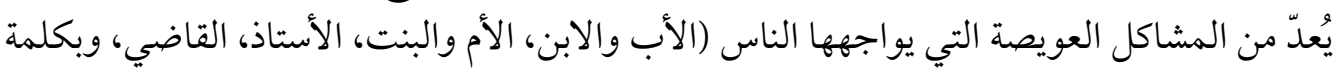

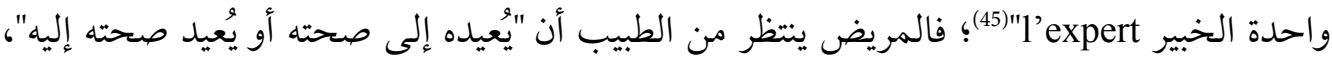

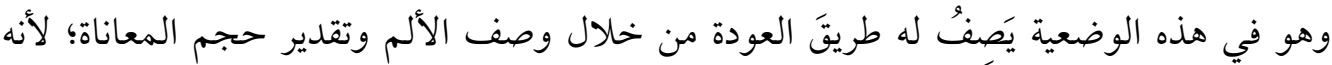

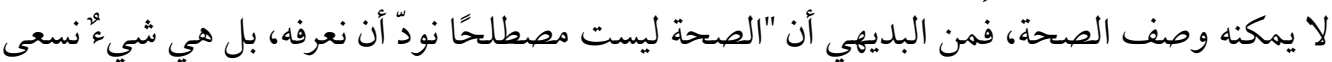

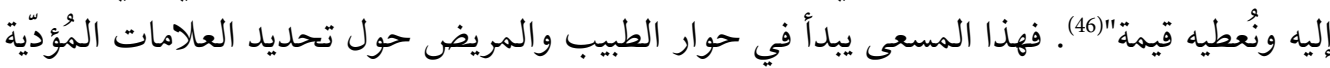

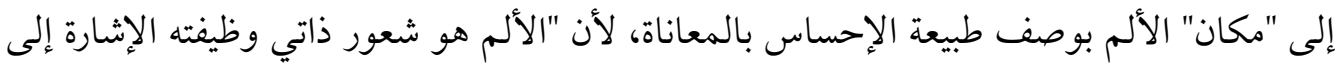

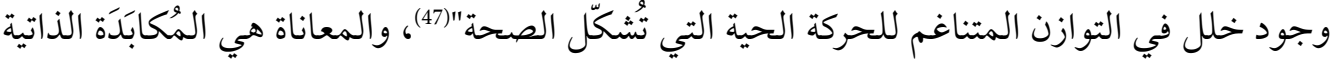

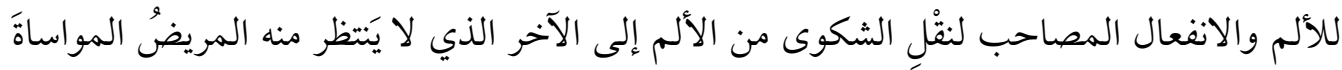

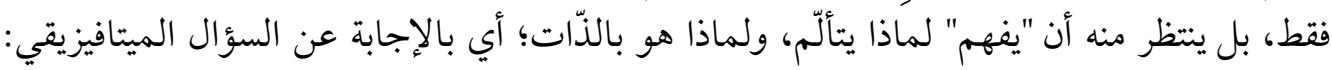

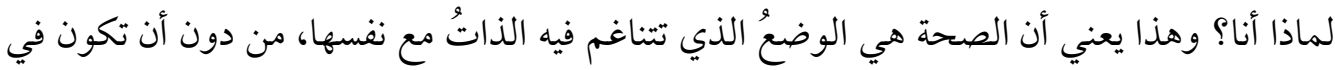

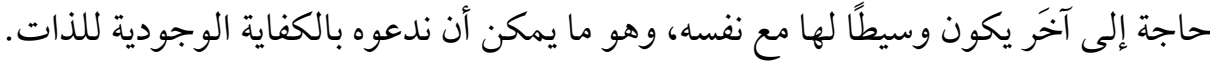

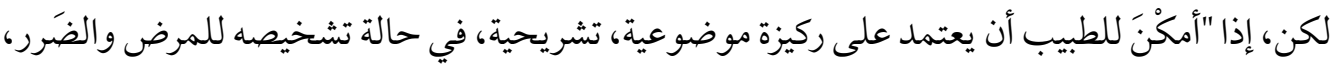

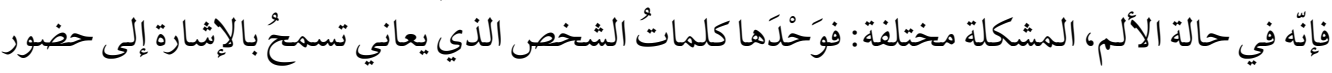

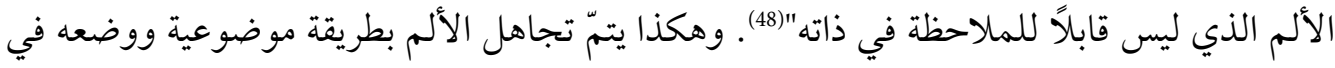

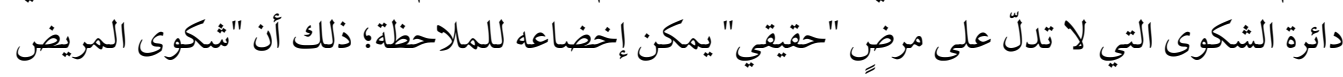

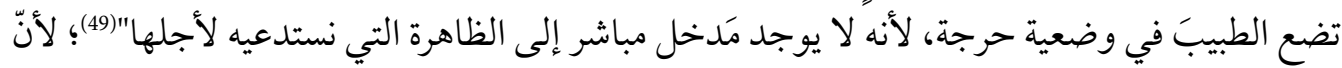

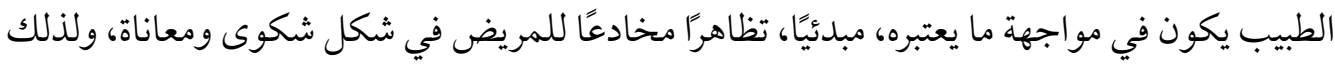

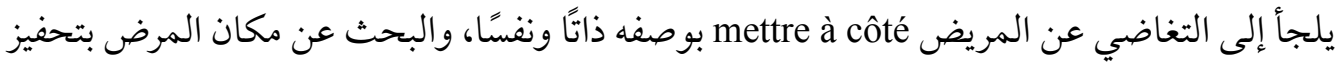

(45) Gadamer, p. 122.

(46) Daniel Callahan, The Roots of Bioethics: Health, Progress, Technology, Death (New York: Oxford University Press, 2012), p. 71.

(47) Gadamer, p. 118.

(48) Rey, p. 18.

(49) Ibid. 
الألم و إثارته في جسد المريض، ولكنه لا مفرّ من الاستدلال عليه بالسؤال الشهير: هل تحسّ بالألم

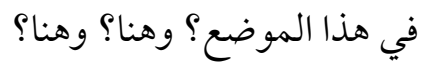

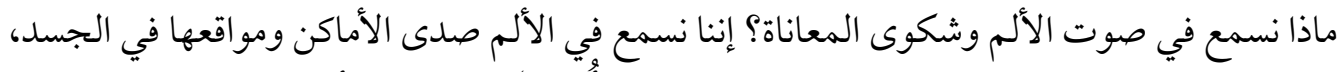

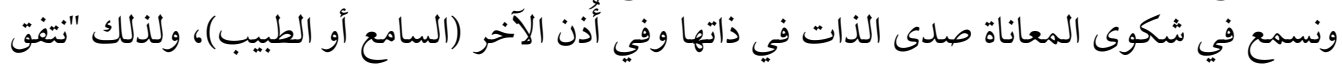

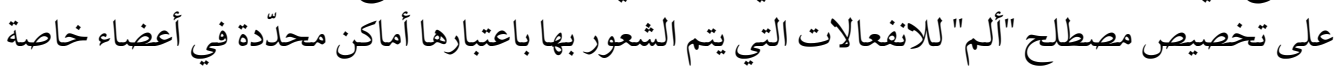

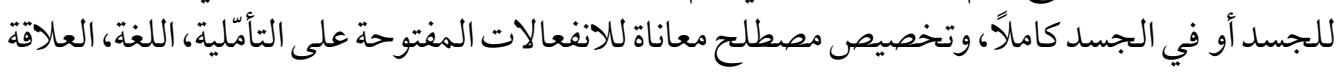

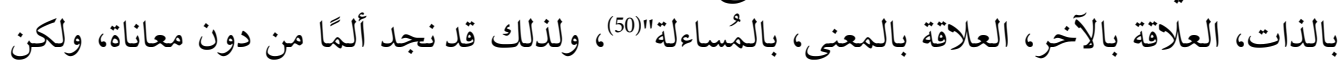

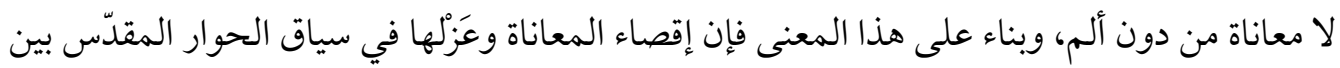

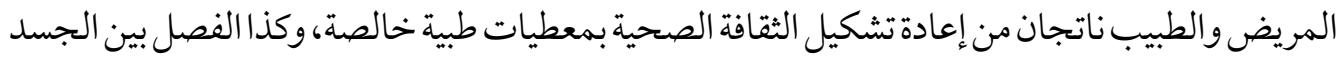

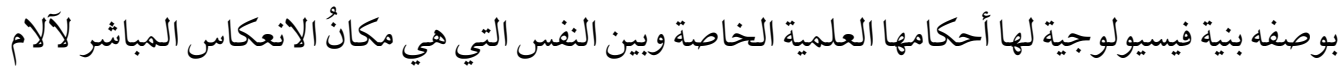

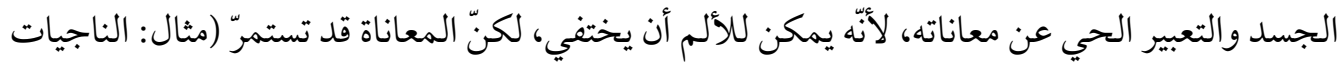

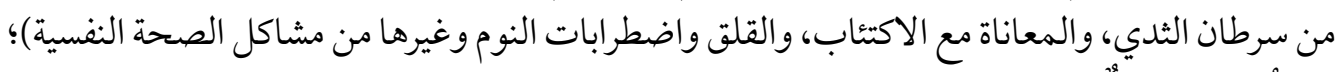

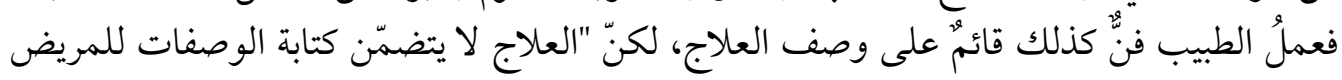

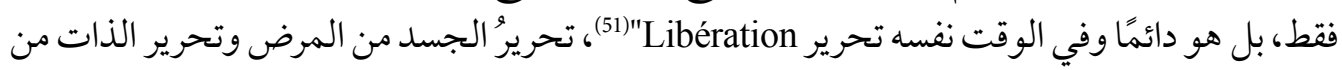

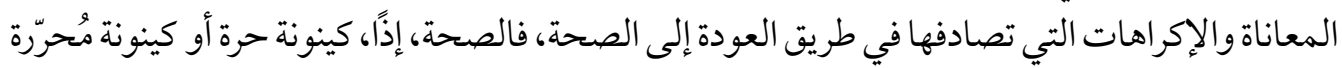

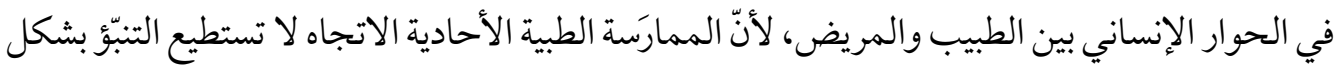

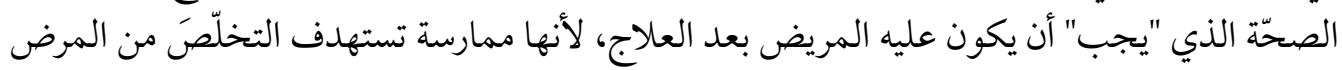
ولا تضع في الحسبان كيفية التخلّص من المعاناة.

أدّى الوثوق المتزايد بأنماط العلاج الطبي المعاصر وتطور آلياته التقنية إلى إخضاع المريض كُّليَّا

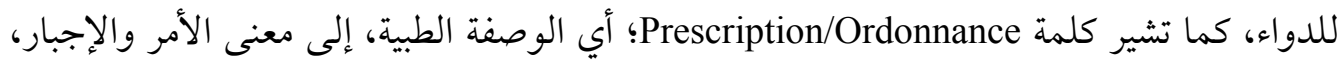

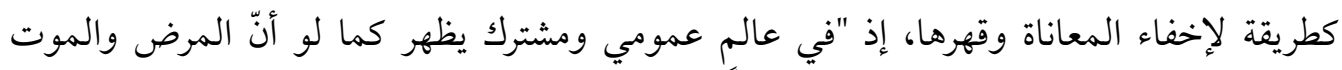

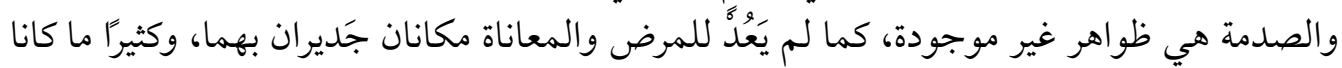

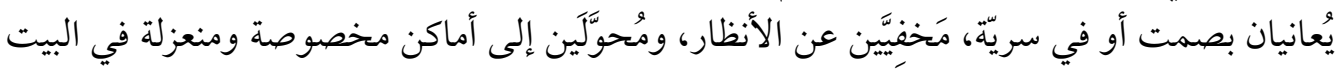

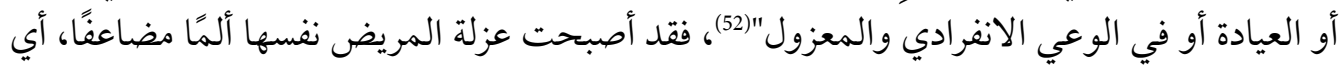

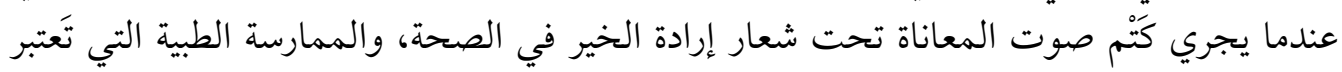

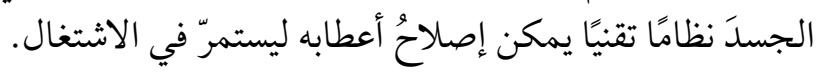

كرّست نظرية كونغيلهام هذا المعنى، أي تلك "النظرية ذات الطابع الموضوعي جداًّا، حيث فيها

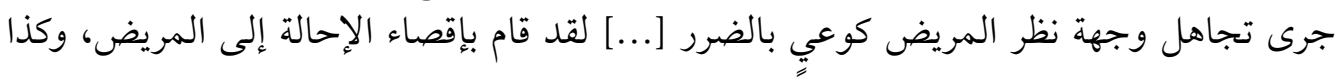

(50) Paul Ricœur, "La Souffrance n'est pas la douleur," in: Souffrance et douleur, autour du Paul Ricour, Claire Marin \& Nathalie Zaccaï-Reyners (eds.) (Paris: Presses Universitaires de France, 2013), p. 14.

(51) Gadamer, p. 119.

(52) Taylor, p. 38. 
تصوّر الحالة المرضية والحالة الفيسيولوجية بوصفهما حالتين متجانستين: فالمرض هو مجرّد

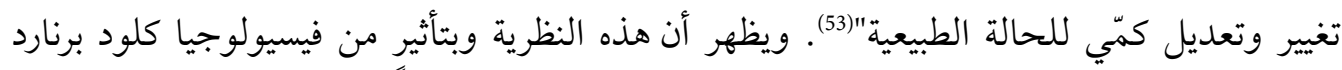
ه Claude Bernard

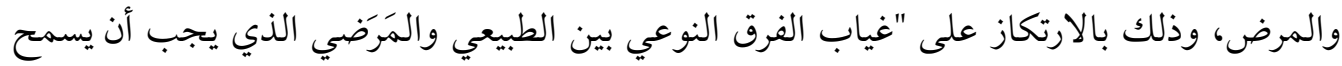

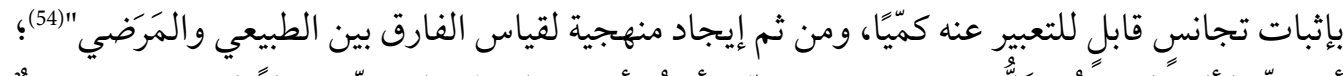

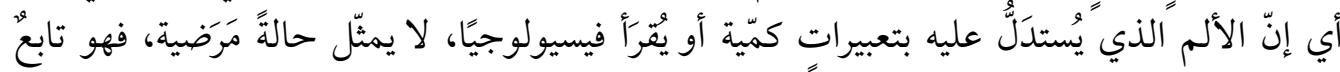

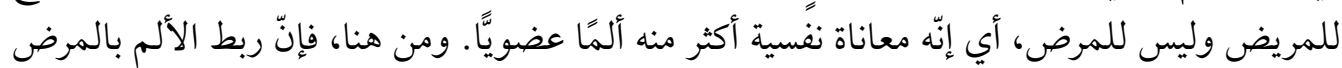

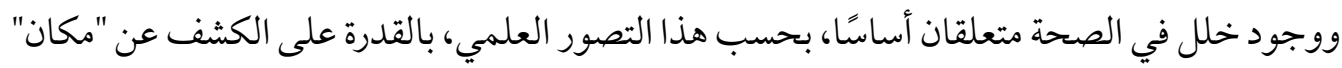

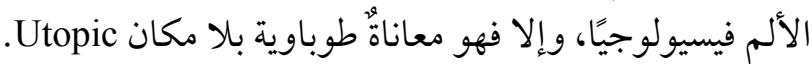

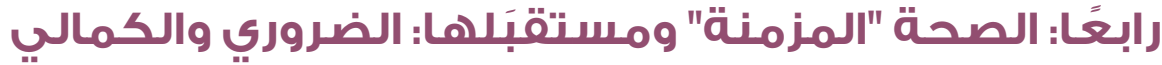

ليست الإيتيقا الطبية وإيتيقا الصحة بالخصوص في صدام مع البنية العلمية للطبّ المعاصر أو من

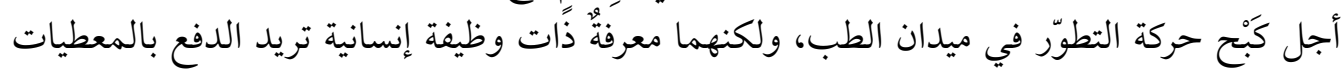
الأنثروبولوجية والأخلاقية للمريض إلى داخل قلب الحوار بين الطبيب والمريض، و وإلى داخل النقاشات

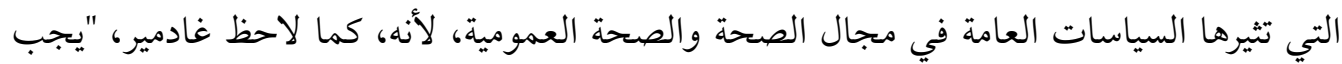

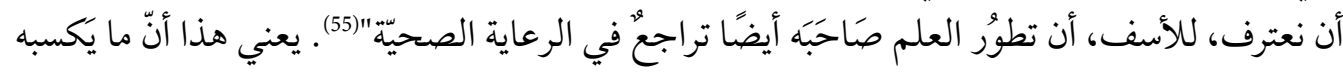

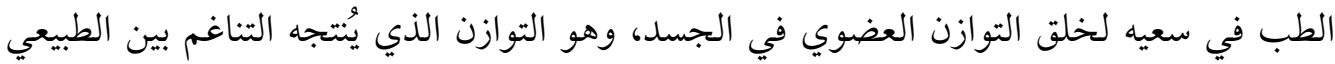

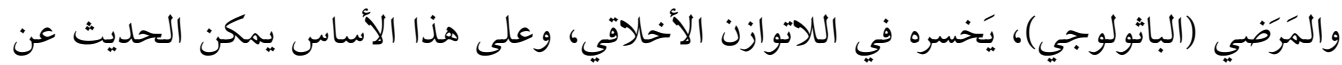

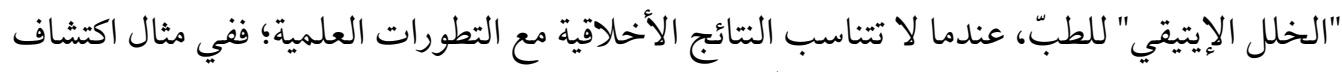
الأصل الجيني لمرض "المنغولية" أو "التثلّث الصبغي 21" Trisomie21 من طرف الإنيفي العالم الفرنسي

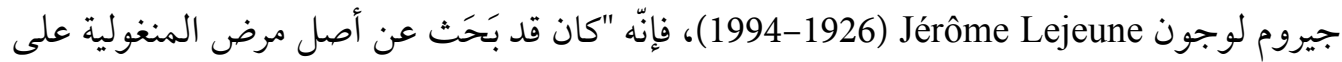

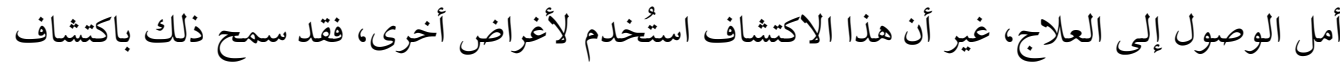

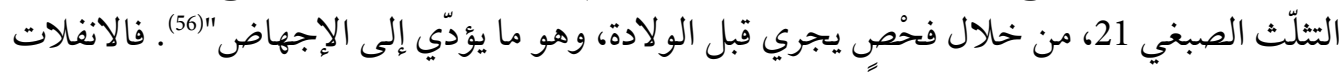

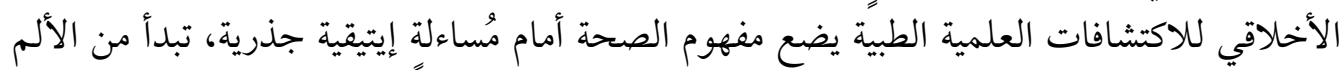

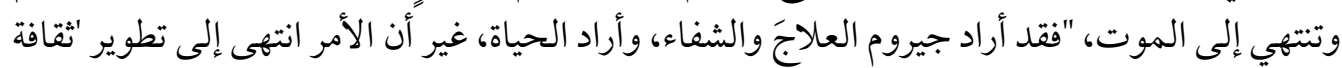

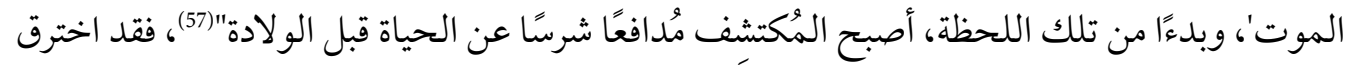

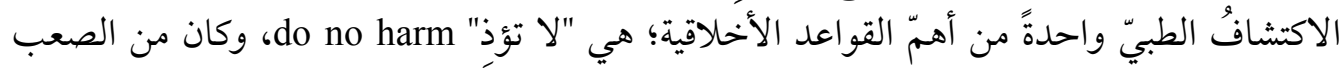

(53) Rey, pp. 7-8.

(54) Ibid., p. 8 .

(55) Gadamer, p. 116.

(56) François-Xavier Putallaz \& Michel Salamolard, Le sens de l'homme au cour de la bioéthique (Saint Maurice: Editions Saint-Augustin, 2006), p. 29.

(57) Ibid., p. 29. 
إصلاحُ ذلك الاختراق إلا أخلاقيًا، أي بإعادة الاعتبار إلى قيمة الحياة قبل الولادة مهما كانت الدوافع العلمية لوأدها. يعمل المشروعُ العلمي للطب المعاصر على محاولة حفظ الصحة ووقايتها وترقيتها، ولكنْ سيتحتّم المقال

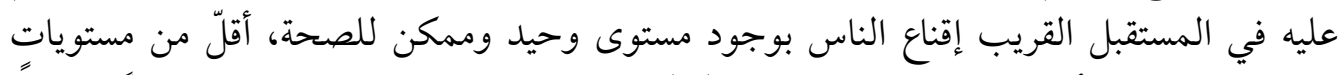

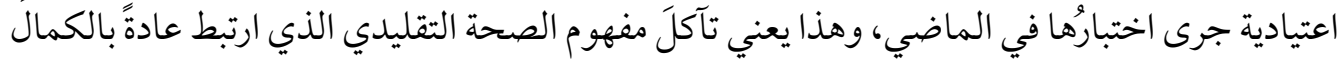

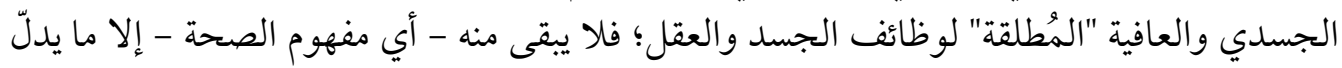

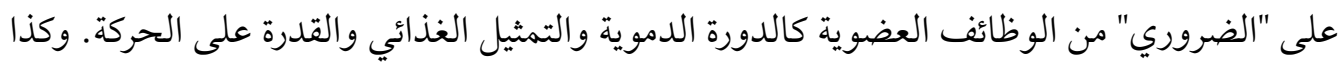

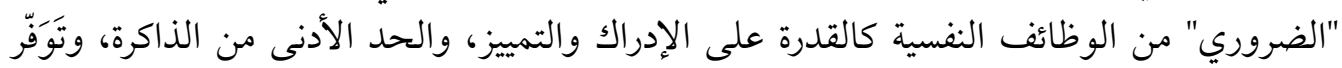

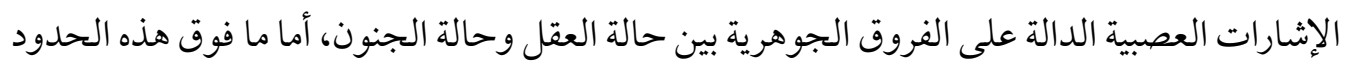

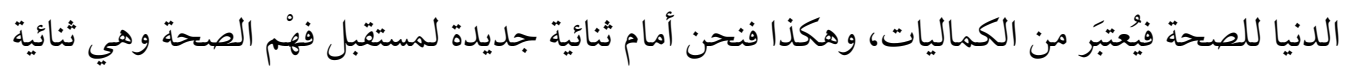

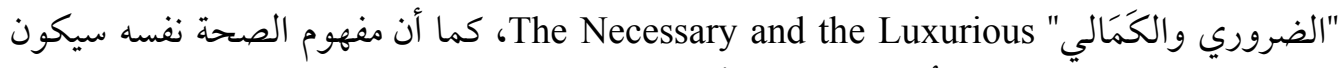

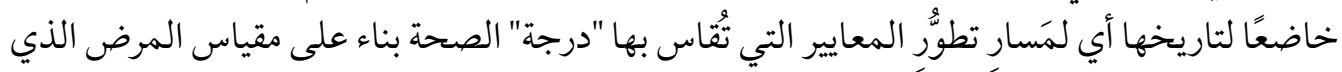

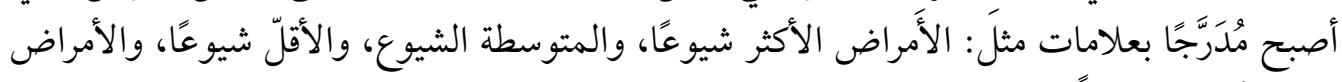
النادرة أو النادرة جدًَا.

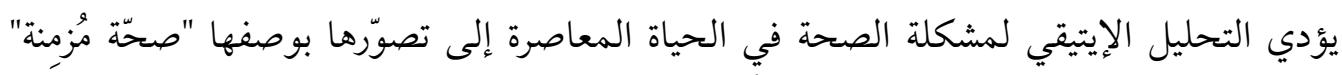

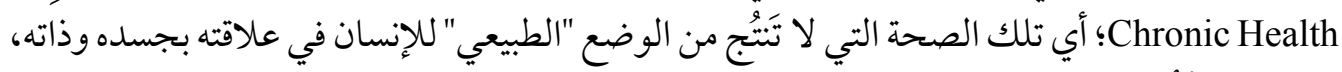

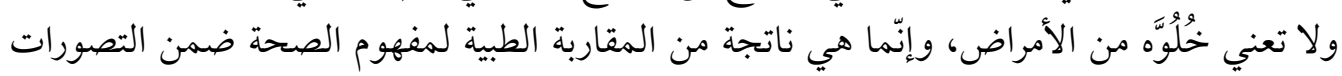

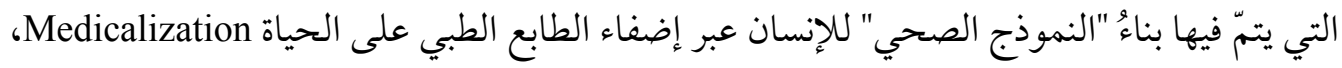

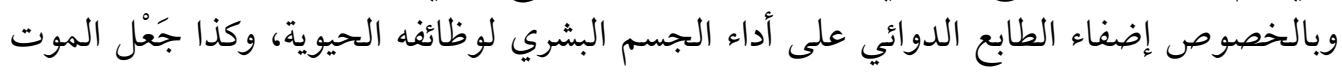

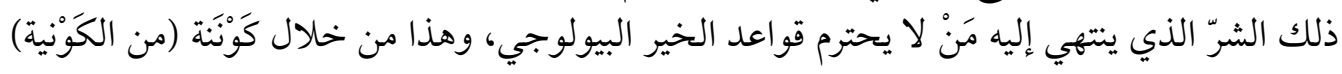
وعنأنrsalization

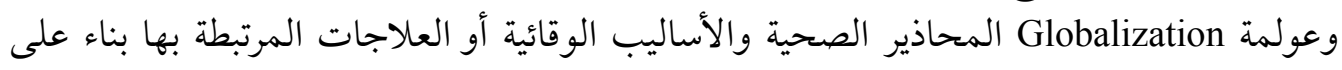

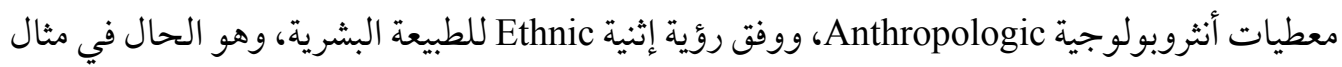

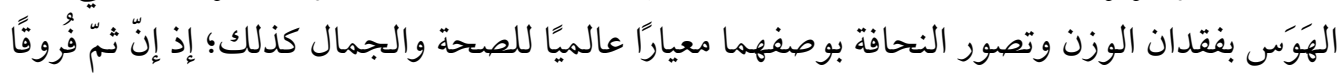

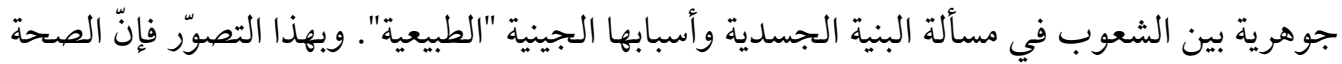

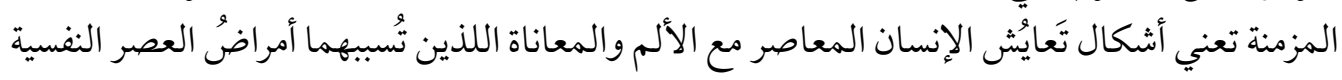

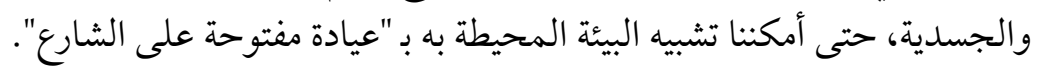

\section{خَاتمة}

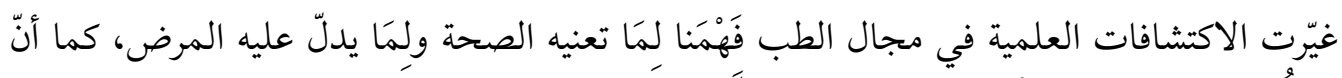

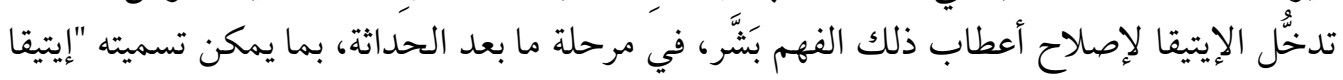

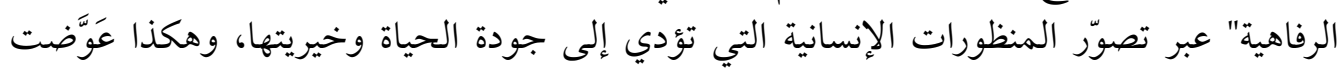


الرفاهيةٌ Well-Being السعادة Happiness أي في تحويل المعياري إلى المعيش ولكنّه المعيش الأكثر

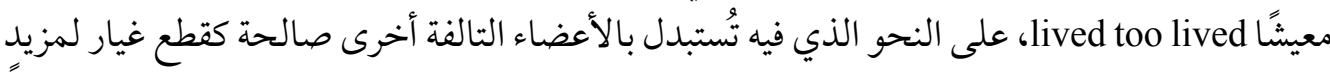

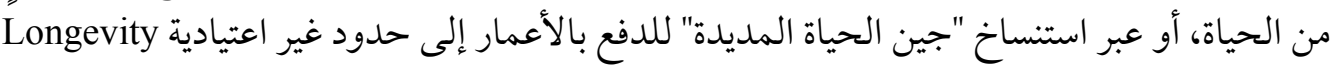

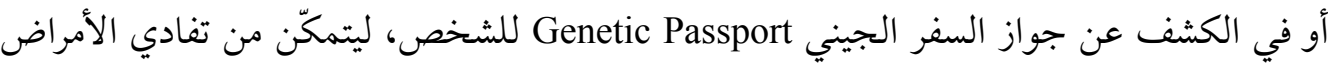

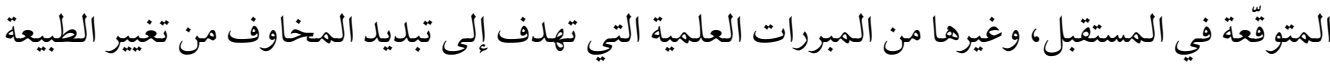

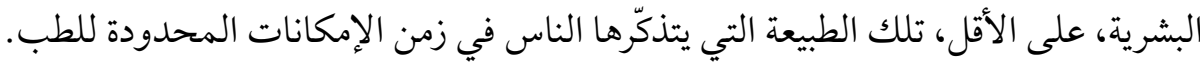

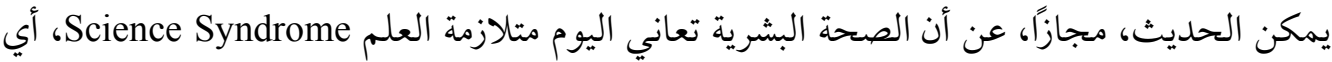

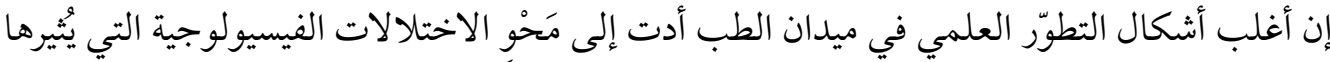

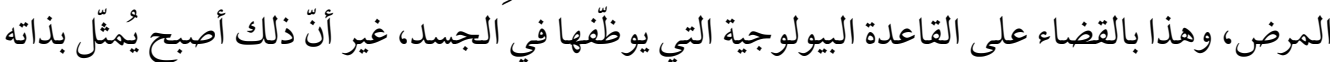

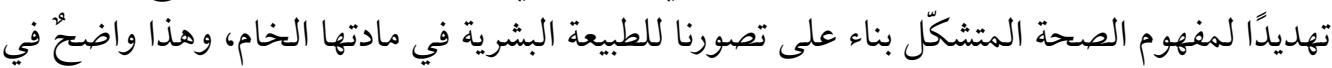

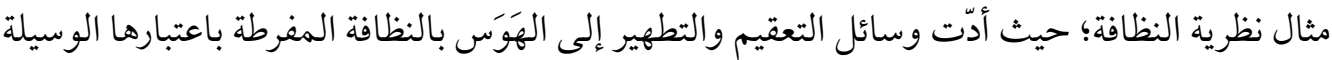

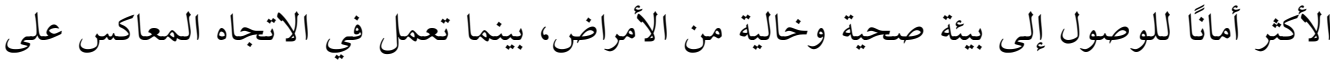

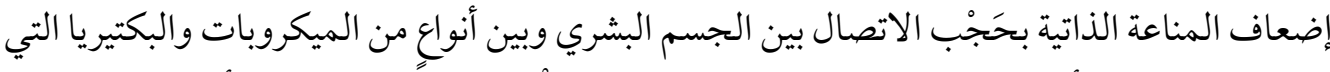

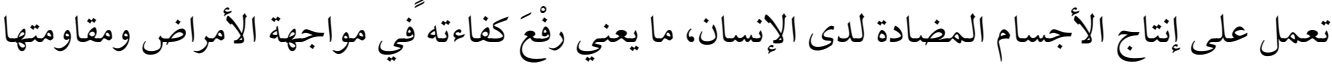

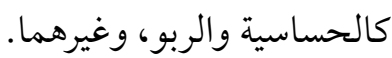

\section{References}

Boitte, Pierre. Éthique, justice et santé: Allocation des ressources en soins dans une population vieillissante. Paris: Artel; Montréal: Fides, 1995.

Boorse. Christopher et al. Meaning and Medicine: A Reader in the Philosophy of Health Care. New York: Routledge, 1999.

Cadoré, Bruno. L'expérience bioéthique de la responsabilité. Paris: Artel; Montréal: Fides, 1994.

Callahan, Daniel. The Roots of Bioethics: Health, Progress, Technology, Death. New York: Oxford University Press, 2012.

Canguilhem, Georges. Le Normal et le Pathologique. 12 $12^{\mathrm{eme}}$ éd. Paris: Presses Universitaires de France, 2013.

Dawson, Angus (ed.). The Philosophy of Public Health. London/ New York: Routledge, 2016.

Ekanga, Basile. Les Fondements éthiques de la Bioéthique. Bloomington: iUniverse LLC, 2013.

Gadamer, Hans-Georg. Philosophie de la santé. Marianne Dautrey (trad.). Paris: Grasset, 1998.

Gimmler, Antje, Christian Lenk \& Gerhard Aumuller. Health and Quality of Life: Philosophical, Medical and Cultural Aspects. Homburg/ London/ Munster: LIT, 2002. 
Guinchaud, Paulette \& Jean François Petit. Une société de soins: Santé, travail, philosophie, politique. Paris: Les éditions de l'Atelier; Les éditions ouvrières, 2011.

Jecker, Nancy S., Albert R. Jansen \& Robert A. Pearlman. Bioethics: An Introduction to the History, Methods, and Practice. Sudbury: Jones and Bartlett Publishers, 2007.

Kottow, Miguel. From Justice to Protection: A Proposal for Public Health Bioethics. New York: Springer, 2012.

Lefèvre, Céline. "De la philosophie de la médecine de Georges Canguilhem à la philosophie du soin médical." Revue de Métaphysique et de morale. vol. 2, no. 82 (2014).

Lewins, Frank. Bioethics for Health Professionals: An Introduction and Critical Approach. Melbourne: Macmillan Education Australia, 1996.

Massé, Raymond. Éthique et santé publique: Enjeux, valeurs et normativité. Québec: Les presses de l'université Laval, 2003.

Méthot, Pierre-Olivier. "Les Concepts de santé et de maladie en histoire et en philosophie de la médecine." Revue Phares. vol. XVI. Université Laval (2016).

Putallaz, François-Xavier \& Michel Salamolard. Le sens de l'homme au cour de la bioéthique. Saint Maurice: Editions Saint-Augustin, 2006.

Rey, Lucie. Qu'est-ce que la douleur? Lecture de René Leriche. Paris: Éditions L'Harmattan, 2010.

Souffrance et douleur, autour du Paul Ricour. Claire Marin \& Nathalie Zaccaï-Reyners. (eds.). Paris: Presses Universitaires de France, 2013.

Svenaeus, Fredrik. The Hermeneutics of Medicine and The Phenomenology of Health: Steps towards A Philosophy of Medical Practice. Berlin/ Dordrecht: Springer Science \& Business Media, 2000.

Taylor, Carol \& Roberto Dell'oro. Health and Human Flourishing Religion, Medicine and Moral Anthropology. Washington, DC: Georgetown University Press, 2006. 